EFFECTS OF MENTAL FATIGUE ON EXERCISE DECISION-MAKING 
M. Sc. Thesis - Sheereen Harris; McMaster University - Kinesiology

\section{EFFECTS OF MENTAL FATIGUE ON EXERCISE DECISION-MAKING}

\section{By SHEEREEN HARRIS}

B. Sc. Specialized Honours Kinesiology \& Health Science

A Thesis Submitted to the School of Graduate Studies in Partial Fulfilment of the Requirements for the Degree Master of Science

McMaster University ( C Copyright by Sheereen Harris, August 2018 
M. Sc. Thesis - Sheereen Harris; McMaster University - Kinesiology

MASTER OF SCIENCE (2018)

(Kinesiology)

McMaster University

Hamilton, Ontario

TITLE: $\quad$ Effects of Mental Fatigue on Exercise Decision-Making

AUTHOR: Sheereen Harris

B. Sc. Specialized Honours Kinesiology \& Health Science (York University)

SUPERVISOR: Dr. Steven R. Bray, Ph.D.

NUMBER OF PAGES: $\mathrm{x}, 65$ 
M. Sc. Thesis - Sheereen Harris; McMaster University - Kinesiology

\begin{abstract}
People's decisions regarding effort-based tasks such as engaging in physical activity depend on the subjective value of the activity: weighing the costs against the benefits (Chong et al., 2016). Exerting cognitive effort while performing one task negatively biases people's decisions to exert effort on subsequent cognitive tasks, suggesting a shift in their subjective valuation of the task due to mental fatigue (Kool \& Botvinick, 2014). Similarly, exerting physical effort negatively biases decisions to further exert effort on a future physical task (Iodice et al., 2017a, Iodice et al., 2017b). The purpose of the present study was to investigate the effect of mental fatigue on people's decisions to engage in an acute bout of exercise and whether the effect of mental fatigue on decision-making was mediated by a benefit-cost analysis. Among those who decided to exercise, the study also aimed to investigate the relationship between mental fatigue and exercise behaviours during a self-selected, self-paced, bout of exercise. Recreationally active participants $\left(N=55, M_{a g e}=19.04 \pm 1.04\right.$ years $)$ completed either a 10minute, high cognitive demand (Stroop) task or low cognitive demand (documentary viewing) task to manipulate levels of mental fatigue. Participants then made a choice between engaging in a 20-minute self-paced moderate-to-vigorous intensity exercise task or a 20-minute non-exercise task. Prior to choosing, participants rated their mental fatigue and their perceived benefits and costs of the exercise task. The cognitive task had a strong effect on mental fatigue $(p<.001$, Cohen's $d=1.40$ ). The mediation analysis showed no direct effect of mental fatigue on choice; however, there was a significant indirect effect indicating the benefit-cost score mediated the effect of mental fatigue on choice (95\% C.I. $=-.02$ to -.0004). Higher levels of mental fatigue were associated with a lower benefit-cost score $(r=-.33, p=.01)$ which, in turn, was associated with a decreased likelihood of choosing the exercise task $(r=.31, p=.02)$. For those who chose
\end{abstract}




\section{Sc. Thesis - Sheereen Harris; McMaster University - Kinesiology}

to engage in the exercise task $(N=28)$, higher levels of mental fatigue were associated with higher ratings of perceived exertion $(r=.38, p=.05)$. Findings provide insight into the effects of mental fatigue on people's exercise behaviours, illustrating a rational decision-making process that is dependent upon the subjective evaluation of the costs and benefits of engaging in physical activity or sedentary alternatives. 
M. Sc. Thesis - Sheereen Harris; McMaster University - Kinesiology

\section{ACKNOWLEDGEMENTS}

I would like to first thank my supervisor, Dr. Steven Bray. Thank you for recognizing the "thinker" in me and encouraging my growth by questioning and challenging me in the most productive ways I could ever imagine. I look forward to learning and growing as a scientist under your guidance. To my committee members, Dr. Jennifer Heisz and Dr. Martin Gibala, thank you for your comments and suggestions throughout this project. Your insight is truly appreciated.

Thank you to my labmates, both past and present for welcoming me so openly into the McMaster kinesiology community and for blurring the line between work and play. It has been an absolute honour working and growing alongside each one of you.

To Kristen \& Michelle. Thank you for helping me navigate the challenges of graduate studies - and life for that matter and for always being my voices of reason. You both keep my days and spirits bright and have helped me maintain my sanity amidst the seemingly insurmountable stresses of graduate school. I appreciate you both ever so kindly.

Last, but certainly not least, to Mom, Dad, and Shari. Thank you for constantly cheering me on at the imaginary finish line in this sport of academia. Your continuous support and encouragement throughout my never-ending journey through higher education has never gone unnoticed. 
SECTION

TABLE OF CONTENTS

TITLE PAGE

PAGE

DESCRIPTIVE NOTE

ABSTRACT

ACKNOWLEDGEMENTS

TABLE OF CONTENTS

LIST OF FIGURES, TABLES, AND APPENDICIES

LIST OF ABBREVIATIONS

DECLARATIONS OF ACADEMIC ACHIEVEMENT

iii

iv

vi

vii

viii

ix

$\mathbf{x}$

\section{INTRODUCTION}

How can we understand decision-making?

What circumstances affect perceptions of costs and benefits?

Self-control, mental fatigue, and decision-making

Statement of the problem

Hypotheses

METHODS

Participants

Tasks and Apparatus

Experimental Manipulations

Measures

Study Design and Procedures

Data Analysis

RESULTS

Data Screening and Assumption Checks

Preliminary Analyses

Primary Analyses

Bivariate Correlations Between Psychological Variables

Indirect (Mediation) Analysis

Mental Fatigue and Exercise Behaviour

DISCUSSION

Mental fatigue and exercise decision-making

Mental fatigue and psychological correlates of decision-making

Mental fatigue and exercise behaviours

STRENGTHS AND LIMITATIONS

FUTURE DIRECTIONS

CONCLUSION

APPENDICIES

REFERENCES

\section{1}

17

17

18

20

21

24

26

29

29

29

31

33

34

35

37

37

38

39

41

44

46

48

57 


\section{LIST OF FIGURES, TABLES, AND APPENDICIES}

\section{FIGURES}

Figure 1. Illustration of M-GXT 19

Figure 2. Frequency distribution of choice $\quad 32$

Figure 3. Frequency histogram of post-cognitive task mental fatigue scores $3 \mathbf{3 2}$

Figure 4. Scatterplot between mental fatigue and exercise benefits-costs $\quad \mathbf{3 4}$

Figure 5. Direct effect of mental fatigue on choice 35

Figure 6. Indirect effect of mental fatigue on choice 35

\section{TABLES}

Table 1. Descriptive statistics of potential covariates and manipulation checks $\quad 30$

Table 2. Bivariate correlations between mental fatigue, exercise benefits, $\quad 33$ exercise costs, and choice

Table 3. Bivariate correlations between mental fatigue, exercise RPE and 36 workload

\section{APPENDICIES}

Appendix A. Physical Activity Readiness Questionnaire $\quad 48$

Appendix B. Ratings of Perceived Exertion 44

Appendix C. Ratings of Perceived Mental Exertion $\quad 50$

Appendix D. Subjective Mental Fatigue Visual Analogue Scale 51

Appendix E. International Physical Activity Questionnaire $\quad 52$

Appendix F. Physical Activity Enjoyment Scale $\quad 53$

Appendix G. Brief Self-Control Scale $\mathbf{5 5}$

Appendix H. Cost-Benefit Measure $\quad 56$ 
M. Sc. Thesis - Sheereen Harris; McMaster University - Kinesiology

\section{LIST OF ABBREVIATIONS}

$\begin{array}{ll}\text { BMI } & \text { Body Mass Index } \\ \text { EBBS } & \text { Exercise Benefits/Barriers Scale } \\ \text { HR } & \text { Heart Rate } \\ \text { IPAQ } & \text { International Physical Activity Questionnaire } \\ \text { M-GXT } & \text { Modified Graded Exercise Test } \\ \text { M } & \text { Mean } \\ m l & \text { Millilitres } \\ \text { MVPA } & \text { Moderate to Vigorous Physical Activity } \\ \text { PACES } & \text { Physical Activity Enjoyment Scale } \\ \text { PAR-Q } & \text { Physical Activity Readiness Questionnaire } \\ \text { RPE } & \text { Rating of Perceived Exertion } \\ \text { RPE-M } & \text { Rating of Perceived Mental Exertion } \\ \text { RPM } & \text { Rotations per Minute } \\ \text { SD } & \text { Standard Deviation } \\ \text { TTM } & \text { Transtheoretical Model } \\ \text { VAS } & \text { Visual Analogue Scale } \\ \text { W } & \text { Watt } \\ d & \text { Cohen's d } \\ r & \text { Pearson's correlation }\end{array}$




\section{DECLARATION OF ACADEMIC ACHIEVEMENT}

\section{Sheereen Harris' role:}

- Amended ethics application at McMaster University

- Designed study protocol and chose measures

- Recruited participants

- Organized visits and set up lab equipment and materials

- Supervised volunteers assisting with data collection

- Responsible for manuscript preparation

\section{Role of co-authors:}

- SB assisted SH with ethics application

- $\mathrm{SB}$ assisted SH with study design and selection of measures

- SB assisted SH with data interpretation

- SB obtained study funding 
M. Sc. Thesis - Sheereen Harris; McMaster University - Kinesiology

\section{INTRODUCTION}

Current physical activity guidelines recommend that adults attain at least 150 minutes of moderate-to-vigorous intensity physical activity (MVPA) each week to accrue physical and psychological benefits (Tremblay et al., 2011). For example, regular MVPA reduces the risk of numerous chronic diseases and disorders such as cardiovascular disease, cancer, osteoporosis, and hypertension (Colley et al., 2011; Gilmour, 2007; Kesaniemi et al., 2001, Pate et al., 1995). Additionally, MVPA leads to increases in positive affect and self-esteem (Szabo, 2003; Wichers et al., 2012). However, data from the Canadian Health Measures Survey shows that only approximately $20 \%$ of Canadian adults are meeting these guidelines (Colley et al., 2011), which poses a major threat to public health and raises questions as to why more Canadians are not active.

Most people possess the physical capabilities to engage in physical activity, yet many barriers (or costs) to physical activity have been identified; including work commitments, other priorities, lack of time, and feeling tired (Salmon, Owen, Crawford, Bauman, \& Sallis, 2003). While many barriers may exist, it is also important to recognize that people make conscious decisions about whether or not to be physically active and despite the many commonly cited benefits of regular exercise, frequently opt for non-physically active alternatives. In order to more effectively promote physical activity to the population, a better understanding of the processes underlying decision-making, specifically pertaining to physical activity behaviours, is needed.

\section{How can we understand decision-making?}

Behavioural economics (behavioural choice theory) 


\section{Sc. Thesis - Sheereen Harris; McMaster University - Kinesiology}

Behavioural choice theory, or behavioural economics, is a theoretical perspective that extends traditional theories of economic decision-making and can be used to explain and predict people's decision-making. Traditional economic theory posits that human beings are rational, unemotional, and have unlimited information processing capabilities (Mullainathan \& Thaler, 2000). The assumption that people are able to effectively process all relevant information in order to objectively evaluate and then make the optimal choice among multiple alternatives ignores any instances of behaviour deviating from this standard. In reality, people frequently make food choices that exceed their necessary caloric intake, spend more money than they had budgeted, and procrastinate about important tasks they need to carry out. These decisions are made despite being fully aware that alternative behaviours are more beneficial to their long-term objectives. Because actual decision-making fails to adhere to the predictions of traditional economic theory; behavioural economics was developed to integrate research from the disciplines of learning, cognitive psychology, decision-making, and economics to better attempt to understand the factors that influence choices between two or more alternatives (Epstein, 1998; Epstein, 2012). In short, behavioural economics increases the explanatory power of traditional economics by factoring in how people think and feel about their decisions (Camerer \& Loewenstein, 2004).

People's decisions regarding physical activity behaviours represent a choice situation wherein behaviour (i.e., the majority of Canadians are not sufficiently active) tends to deviate from what the available information would suggest aligns best with long-term outcomes (being physically active promotes better health). Epstein (1998) discusses several principles of behavioural economics and how they relate to decision-making when choosing between being physically active or being sedentary. The first principle, "choice of an alternative depends on 


\section{Sc. Thesis - Sheereen Harris; McMaster University - Kinesiology}

the behavioural cost” (p. 258) implicates cost as an important factor in decision-making between two behaviours that differ in at least one evaluative respect. In terms of making a choice between being physically active or sedentary, an important consideration is that physical activity is more costly in terms of energy expenditure or effort requirements in comparison to not engaging in physical activity. The second principle states: "the choice and reinforcing value of a commodity depends on the available alternatives" (p. 258). Again, in terms of physical activity decision-making, an undergraduate student may opt out of exercising to engage in a (highly valued) leisurely social activity with friends, but be more likely to exercise when the most salient alternative option is studying for a test or writing a paper. The first two principles relate to the extent to which decisions are guided by fluctuations in the perceived value of exercise due to the available alternatives. Conversely, the third principle states that "choice is important to motivate people to obtain a reinforcer" (p. 259), suggesting that in order for an intentional behaviour change to occur, a person must perceive that they have initiated the activity as opposed to being forced to perform the behaviour. Lastly, the fourth principle states: "choice depends in part on the delay between choosing and receiving the alternative" (p. 259). This principle relates to physical activity as many of the benefits people perceive they stand to gain from being active are temporally delayed from the execution of the behaviour, contrasted with the benefits of being sedentary which are felt almost immediately (e.g., feeling more relaxed). Collectively, these principles highlight the complexities of choice as well as the many components that factor into decision-making and help explain why the majority of the population does not exercise regularly.

\section{Cost-benefit analysis}




\section{Sc. Thesis - Sheereen Harris; McMaster University - Kinesiology}

Although behavioural economics offers a comprehensive set of principles that are proposed to guide people's decision-making, another way that decision-making about physical activity or exercise can be understood is by looking at someone's motivation towards effortful behaviours. Chong, Bonnelle, and Husain (2016) define motivation as "that process which facilitates overcoming the cost of an effortful action to achieve the desired outcome" (p. 72) and state that our motivation for effort-based decisions can be understood as a series of cost-benefit valuations. These valuations involve a trade-off between the costs (e.g., the effort requirements) against the benefits (e.g., anticipated reinforcement or reward) of any particular action, in which a person seeks to maximize benefits while minimizing costs (Chong et al., 2016). Additionally, the cost-benefit approach to motivation accounts for individual differences by parsing apart the unique components that factor into any person's choice regarding potentially effortful behaviours.

Conceptualizing decision-making as a subjectively-evaluative process aimed towards the maximization of benefits and minimization of costs associated with a particular action has relevance to understanding physical activity behaviours. In other words, a person's decisional balance between their perceived advantages or disadvantages of the behaviour provides information related to their effort-based motivations. The transtheoretical model (TTM) of behaviour change (Prochaska \& DiClemente, 1983) has been widely used in the physical activity and exercise domains to categorize people into one of five stages of intentional behaviour change based on their decisional balance (see Marshall \& Biddle, 2001 for meta-analysis). According to the model, people move progressively through stages of behaviour change from Precontemplation (no intention of becoming physically active), to Contemplation (thinking of becoming physically active within the next 6 months), Preparation (making small changes in 


\section{Sc. Thesis - Sheereen Harris; McMaster University - Kinesiology}

behaviour but are still not meeting the criteria of physical activity), Action (meeting the criteria of physical activity but only within the past 6 months), and Maintenance (meeting the criteria for physical activity for 6 months or longer) (Marshall \& Biddle, 2001).

Using the TTM, Marcus and Owen (1992) examined the relationship between people's cost-benefit analysis and their habitual exercise behaviours. Participants $(N=1093)$ filled out a questionnaire assessing their evaluations of the "pros" and "cons" of exercising as well as their current exercise behaviours, the latter of which was used to categorize them into one of the TTM stages of change. People in different stages of change had significantly different scores on the "pro" scale, the "con" scale, and an overall cost-benefit score (benefit score minus cost score). Consistent with the TTM predictions, results showed that the "Precontemplators", who engaged in no physical activity, reported the lowest scores on the "pro" scale, while those in the Maintenance stage scored the highest. The reverse pattern emerged for scores for "Precontemplators" and "Maintainers" on the "con" scale. A similar pattern was evident with the overall benefit-cost score, where those not engaging in the behaviour ("Precontemplators" and "Contemplators") had negative scores reflective of greater perceived costs than benefits whereas people within the Preparation, Action, and Maintenance stages had positive scores that increased in magnitude as the stages progressed. These findings highlight that people closer to engaging in physical activity (i.e., Preparation stage) or regularly engaging in physical activity (i.e., Action and Maintenance stages) perceive more benefits and less costs of exercise.

Findings from Marcus and Owen (1992) demonstrate that there is a relation between people's cost-benefit analyses of physical activity and their exercise behaviours. However, their data are limited insofar as it relates to general patterns of behaviour and not the decisions people make about whether to engage in physical activity or not. Further, although those in the later 
M. Sc. Thesis - Sheereen Harris; McMaster University - Kinesiology

stages of behaviour change more frequently choose to engage in physical activity, understanding one's general perceptions does not account for any momentary physiological or psychological factors that may affect perceptions of the costs or benefits of exercise, and bias decision-making about whether to engage in physical activity one way or the other.

\section{What circumstances affect perceptions of costs and benefits?}

In behavioural and economic research, studies have investigated the relationship between environmental factors and the perceived value of an object or reward. The term "discounting" refers to a phenomenon in which decreases in the subjective value of a reward are observed as the specific factor upon which the reward is contingent increases (Ostaszewski, Bąbel, P., \& Swebodziński, 2013). Two types of discounting have received considerable attention in the literature: delay and effort discounting.

Delay discounting refers to decreases in the subjective value of a reward with an increased time delay of obtaining the reward (Du, Green, \& Myerson, 2002; Johnson \& Bickel, 2002; Kirby \& Maraković, 1996; Madden, Begotka, Raiff, \& Kastern, 2003). Studies examining delay discounting measure how much less an object is worth when one has to wait longer periods of time to receive it, such as when a lesser lump sum payment is offered upfront, rather than a larger payment spread out over time. A relevant study found incremental decreases in the subjective value of a $\$ 10$ reward for delays ranging from six hours to one year, where longer delays caused participants to value the reward progressively less (Madden et al., 2003).

Effort discounting refers to decreases in the subjective value of a reward as the effort expenditure necessary to obtain the reward increases (Hartmann, Hager, Tobler, \& Kaiser, 2013; Ostaszewski et al., 2013, Sugiwaka \& Okouchi, 2004). In an illustrative study, Ostaszewski et 


\section{Sc. Thesis - Sheereen Harris; McMaster University - Kinesiology}

al. (2013) offered participants a hypothetical reward of approximately $\$ 32$ that was contingent on climbing sets of stairs up to the third, 10th, 40th, or 100th floor of a building. It was found that participants perceived the subjective value of the reward to be lower when they were required to climb more stairs in order to obtain the reward.

Hsu and Vlaev (2014) used a discounting paradigm to examine how the subjective value of a reward varies as a function of the energy expenditure and time required for an activity. In their study, participants $(N=166)$ were provided with the hypothetical situation in which they had won a lottery prize and arrived at a designated lottery centre to claim their money. Participants then had to choose between a smaller fixed reward that they could receive immediately or a greater reward they could receive after 15 minutes of engaging in activities requiring different amounts of energy expenditure: waiting for the reward while seated, waiting for the reward while standing in line, or walking to a different lottery centre. It was found that when the reward required 15 minutes of waiting, participants preferred the immediate smaller reward. The strongest preference for the smaller, immediate reward was when the delayed reward required walking for 15 minutes, reflecting a decrease in the subjective value of the reward with increased physical effort requirements. Further, when participants were presented with similar scenarios that involved sitting, standing, or walking for 30, 60, or 120 minutes, greater reductions in the subjective value of the reward were observed at each level. Again, walking was the least preferred activity. While this study demonstrates a discounting effect for both effort and time, the situational states of the participants while making these decisions was unknown, which may have contributed to their valuation of the reward. Thus, further work has examined how different psychological and physiological states may vary from day-to-day or even moment-to-moment and affect these factors. 


\section{Sc. Thesis - Sheereen Harris; McMaster University - Kinesiology}

\section{Decision-making and effort in physically-demanding tasks}

Physical fatigue, or prior exposure to a physically demanding task, is one factor that influences effort and reward perceptions. In a recent study that examined the effects of fatigue on effort-based decision-making, Iodice and colleagues (2017a) trained rats to a point where they knew one end of a symmetrical T-maze contained a high reward $(0.1 \mathrm{ml}$ sweetened condensed milk) and the other a low reward $(0.03 \mathrm{ml}$ sweetened condensed milk) and the rats would consistently choose the high-reward pathway. They then released the rats into the T-maze with the high reward arm re-engineered so it required them to climb over a 10-centimeter barrier in order to obtain the higher reward, while the low reward arm had no obstacle. The rats performed eight trials every day for three days in which they made decisions between the high effort-high reward and low effort-low reward options in separate non-fatigued and physically-fatigued conditions. When non-fatigued (i.e., the rats simply were released into the T-maze after being placed on a mechanical treadmill for 40 minutes at a speed less than normal walking speed), the rats preferred the high effort-high reward option, choosing it on average seven times out of eight on each of the three days. However, when fatigued (i.e., the rats previously ran on a mechanical treadmill at $80 \%$ of their maximal power for 40 minutes), the rats chose the high effort-high reward option significantly less frequently than when non-fatigued (i.e., three times out of eight).

To further investigate that this behaviour reflected a change in decision-making, rather than either the rats' inability to climb over the barrier or insensitivity to the size of the reward, an additional condition of the experiment was introduced. In this condition, an identical 10centimeter barrier was also placed on the low reward arm, resulting in a choice between a high effort-high reward arm and a high effort-low reward arm. After standardizing the required effort 


\section{Sc. Thesis - Sheereen Harris; McMaster University - Kinesiology}

level to attain the rewards between options, the rats regained their preference for the high efforthigh reward arm choosing it on nearly all trials. These findings support an interpretation that physical fatigue alters the cost-benefit computations involved in the evaluation of effort and biased rats' decision-making (Iodice et al., 2017a).

In another study by the same researchers, Iodice and colleagues (2017b) used an effortdiscounting paradigm to examine the effects of prior physical fatigue on the subjective value of a subsequent bout of exercise in human participants. Each participant $(N=20)$ completed two experimental sessions, one consisting of 40 minutes of rest, and the other consisting of 40 minutes of cycling at a workload corresponding to approximately $70 \%$ of their $\mathrm{VO}_{2}$ max. Both experimental sessions were followed by a task in which they responded to a series of choices between exerting no further physical effort or cycling at $70 \%$ of their $\mathrm{VO}_{2 \max }$ for $10,15,20,25$, 30 , or 40 minutes. Participants were offered $15 €, 20 €, 25 €, 30 €, 35 €$, or $40 €$ for each duration of exercise or $10 €$ for not exerting any further physical effort. There were 36 possible pairs to choose from (e.g., " $10 €$ for no effort or $40 €$ for 15 minutes cycling?”) and each pair was repeated 10 times. Participants were asked to choose between the "lesser effort" or "greater effort" options and the subjective value of the reward was calculated using the point of subjective equivalence between the options for each duration. The point of subjective equivalence reflected the monetary value of the reward at which participants selected the effortful and non-effortful options at an equal frequency. For example, if participants selected both options at an equal frequency ( $50 \%$ of the time) when offered $30 €$ for the effortful option (compared to $10 €$ for the non-effortful option), $30 €$ would represent the point of subjective equivalence for that duration. Furthermore, a higher point of subjective equivalence value reflects a larger amount of money 


\section{Sc. Thesis - Sheereen Harris; McMaster University - Kinesiology}

needing to be offered to select the "greater effort" option as frequently as the non-effortful option, indicative of a lower subjective value.

Results of Iodice et al.'s (2017b) study showed an overall effect revealing a decrease in the subjective value of the reward as effort requirements increased (i.e., people needed to be offered progressively larger rewards to engage in the cycling task for longer durations). Further, when comparing the discounting rates between the rested and fatigued conditions, when people were fatigued, the discounting effects were enhanced such that they reported a lower subjective value of the reward at each cycling duration compared to their responses when they were rested. These findings indicate that when people are fatigued, the perceived cost of exerting further effort increases in such a way that a greater reward is necessary for them to choose to perform a more effortful task.

\section{Decision-making and effort in cognitively-demanding tasks}

Cognitively-demanding tasks are effortful and also show evidence of effort-based discounting (Ostaszewski et al., 2013). That is, in a manner similar to that in which physical fatigue can be induced by physical exertion, cognitive exertion can also lead to feelings of fatigue (Inzlicht, Shenhav, \& Oliviola, 2018; McGuire \& Botvinick, 2010) and show similar effort-discounting effects and biasing of decision-making to those seen for physically effortful tasks.

Two experiments conducted by Kool and Botvinick (2014) help illustrate how previous exertion of cognitive effort affects decisions related to the exertion of further cognitive control. Each experiment involved two sessions in which participants had to perform a self-determined combination of two cognitive tasks involving the presentation of a series of photos. The low 


\section{Sc. Thesis - Sheereen Harris; McMaster University - Kinesiology}

cognitive demand task involved simply pressing a button when a photograph of a child was presented. The high cognitive demand task required participants to press a button when the face presented in the current photograph was identical to the face presented three photos earlier. The participants freely chose how they wanted to allocate their time between both tasks throughout the 40-minute sessions and received a reward for each high cognitive demand trial selected. In Experiment 1, Session 1, participants were offered a baseline reward for selection of the high cognitive demand task. In Session 2, a 50\% reduction in the reward from Session 1 was imposed (i.e., when the high demand task was selected during the second session, participants earned half of what they did in the first session). In Experiment 2 the reward scenario was reversed. That is, in Session 1, participants earned a smaller baseline reward for selection of the high cognitive demand task than what was offered in the first experiment, however, this reward was doubled (rather than halved) in Session 2.

Results from Experiment 1 demonstrated that participants increased selection of the low cognitive demand option when the reward was reduced in the second session compared to the first. In contrast, Experiment 2 showed that when the reward was doubled in the second session, selection of the high cognitive demand task increased, demonstrating a greater preference for the more effortful option when a larger reward was offered (Kool \& Botvinick, 2014). However, since decisions were made after either a wage increase or a wage decrease, the context in which one chose to exert cognitive effort in the second session in relation to the first session differed between experiments. Notably, participants who experienced the reward increase in the second session may have assigned a greater value to the reward and perceived a lower cost of the cognitive effort. Consequently, these people may have been more motivated to exert cognitive control (compared to those who experienced the reward decrease) due to perceiving a lower cost 


\section{Sc. Thesis - Sheereen Harris; McMaster University - Kinesiology}

of cognitive effort despite the task being the same. More so, the authors argue that the cost of exerting cognitive control is affected by previous cognitive control exertion. Specifically, when more cognitive control has been previously exerted, or perceived to have been exerted, exerting further cognitive control is more costly leading to withdrawal from subsequent tasks requiring cognitive effort when given a choice. The authors concluded that these differences in participants' perceived costs of cognitive effort and choices of how to allocate their effort might be due to differences in the subjective cost of cognitive control based on the different contexts in which the reward was evaluated (i.e. after a wage increase or decrease). In this way, cognitive effort appeared to be more costly in the context of perceiving to have exerted more cognitive effort in the first session, which may cause people to avoid future activities requiring high exertion demands. Results from these experiments suggest that decisions regarding the exertion of cognitive control require a similar cost-benefit trade-off to those seen for physical effort and that previous exertion of cognitive control may lead to withdrawal from subsequent tasks requiring cognitive effort.

Together, the aforementioned studies demonstrate the flexibility of the cost-benefit analysis. More so, the negative carryover effects of both physical and cognitive exertion on performance of subsequent tasks of the same domain are observed. However, it remains unclear whether effortful exertion in one domain (e.g., cognitive exertion) affects the cost-benefit analysis of a behaviour in a different domain (e.g., physical exertion).

\section{Self-control, mental fatigue, and decision-making}

Research examining the effects of effortful exertion on subsequent performance outcomes across different domains has been undertaken in the area of self-control using the self- 


\section{Sc. Thesis - Sheereen Harris; McMaster University - Kinesiology}

control strength or "ego-depletion" model. Self-control refers to the capacity for controlling or altering one's actions or responses in order to align with specific standards or goals (Baumeister, Vohs, \& Tice, 2007; Muraven, Tice, \& Baumeister, 1998). It has been proposed that people's abilities to exert self-control over their thoughts, emotions and behaviours are governed by a limited resource (Muraven et al., 1998), such that utilizing some self-control while performing one task leads to feelings of fatigue and a reduced ability to exert future self-control on subsequent tasks. In line with this idea, research has consistently shown that performing a task that requires self-control leads to performance impairments on subsequent tasks that also involve self-control even when the tasks performed are from different domains. For example, exerting self-control over emotions has been found to impair performance of self-regulated physical exercise (Wagstaff, 2014). However, the limited resource model has been criticized for a lack of theoretical development articulating what the "limited" resource actually is (Friese, Loschelder, Gieseler, Frankenback, \& Inzlicht, 2018; Inzlicht \& Berkman, 2015; Inzlicht \& Marcora, 2016). More recent conceptualizations suggest that self-control represents the value-based choice between an action consistent with a goal or an alternative action that is less-consistent or inconsistent with one's goals (Berkman, Hutcherson, Livingston, Kahn, \& Inzlicht, 2017). What is common to both perspectives is the proposition that the exertion of self-control requires conscious effort, which over time can lead to feelings of fatigue (Hagger, Wood, Stiff, \& Chatzisarantis, 2010). Given that fatigue is commonly cited as a barrier to physical activity (Salmon et al., 2003), it is essential for research to investigate how being in a state of fatigue affects decision-making processes pertaining to physically active behaviours that require selfcontrol or self-regulation. 


\section{Sc. Thesis - Sheereen Harris; McMaster University - Kinesiology}

\section{Decision-making when cognitively-demanding tasks precede physically-demanding tasks}

To the best of our knowledge, no studies have assessed how mental fatigue affects the cost-benefit analysis of a subsequent physical task. Mental fatigue is a psychobiological state experienced during or following prolonged and challenging cognitive activity, which manifests as tiredness, exhaustion and decreased motivation (Boksem, Meijman \& Lorist, 2006; Boksem \& Tops, 2008). When mentally fatigued, people frequently report higher perceptions of exertion while performing physical tasks (e.g., Marcora, Staiano, \& Manning, 2009; Salam, Marcora \& Hopker, 2018; Zering, Brown, Graham, \& Bray, 2016), and exhibit performance decrements, which manifest as: shorter time to exhaustion when performing physical tasks at a fixed workload (Brown \& Bray, 2017b; Marcora et al., 2009), increased time to complete a fixeddistance time trial (MacMahon, Schücker, Hagemann, \& Strauss, 2014), and reductions in selfselected power output (Brownsberger, Edwards, Crowther, \& Cottrell, 2013).

Given the deleterious effects mental fatigue has on exercise performance, recent work has examined whether mental fatigue affects psychological perceptions prior to initiating a bout of exercise, which may mediate subsequent performance decrements. Some studies have found that cognitive control exertion leads to lower ratings of positive affect (Brown \& Bray, 2017a; MacMahon, Schücker, Hagemann, \& Strauss, 2014) and reductions in task-self-efficacy (Brown \& Bray, 2017a; Graham \& Bray, 2015; Graham, Martin Ginis, \& Bray, 2017). Conversely, other studies have observed no differences in variables such as task motivation (Brown \& Bray, 2017b; Brown \& Bray, 2018; MacMahon et al., 2014; Zering et al., 2016) or mood (Brownsberger et al., 2013) following cognitive control exertion.

A recent study by Brown and Bray (under review) measured specific psychological factors preceding a 20-minute bout of self-paced exercise at a vigorous intensity. Participants 


\section{Sc. Thesis - Sheereen Harris; McMaster University - Kinesiology}

reported their goal commitment and intended exercise intensity for the exercise task before and after performing mentally fatiguing and non-mentally fatiguing tasks. Results showed a significant reduction in both psychological variables when participants were mentally fatigued. Similar reductions in intended exercise intensity when people are mentally-fatigued have been reported in other studies involving aerobic exercise (Brown \& Bray, 2018) as well as resistance exercises (Martin Ginis \& Bray, 2010) suggesting that people plan to invest less effort on exercise tasks when mentally-fatigued. However, findings are limited in the sense that the mechanisms underlying these intentional changes have not been assessed. One potential mechanism explaining these changes in intentions could be changes in people's subjective valuation of the behaviour, specifically through cost-benefit evaluations. However, to our knowledge, changes in perceived benefits and costs related to exercise and the effects of this evaluation on decisions to engage in exercise have not been investigated.

\section{Statement of the problem}

The purpose of the present study was to investigate the effects of mental fatigue on people's decisions to engage in an acute bout of MVPA and whether the relationship between mental fatigue and acute decision-making is mediated by a cost-benefit analysis. Additionally, the study aimed to evaluate the relationship between mental fatigue and exercise behaviours during a self-selected self-paced bout of exercise among participants who choose to exercise. To these ends, participants completed either a high cognitive demand task intended to increase their perceptions of mental fatigue (Brown \& Bray, 2017a; Shortz \& Mehta, 2017; Vrijkotte et al., 2018), or a low cognitive demand task designed to maintain fatigue levels. Following each manipulation, they were given a choice between engaging in a 20-minute self-paced moderate- 


\section{Sc. Thesis - Sheereen Harris; McMaster University - Kinesiology}

to-vigorous intensity exercise task or a 20-minute non-exercise task. Prior to making their choice, they reported their perceived benefits and costs of the exercise activity and then selected and engaged in the activity of their choosing.

\section{Hypotheses}

It was hypothesized that people who were mentally-fatigued would choose the exercise option less frequently than those who were not mentally-fatigued. Additionally, it was hypothesized that mental fatigue would be associated with people's benefit-cost ratings for the exercise option such that greater mental fatigue would be correspondent with greater perceived costs of exercise. Benefit-cost ratings were hypothesized to be associated with choice of the exercise/non-exercise tasks, such that higher perceived costs would be correspondent with a lower frequency of choosing to exercise. It was also hypothesized that mental fatigue would indirectly affect choice through a causal process mediated by a benefit-cost analysis. Lastly, it was hypothesized that, among the participants who chose the exercise option, people who were mentally-fatigued would have poorer physical performance, measured as lower self-selected workloads during the 20 -minute bout of exercise. 
M. Sc. Thesis - Sheereen Harris; McMaster University - Kinesiology

\section{METHODS}

\section{Participants}

Participants were 55 ( $n=27$ women) recreationally-active university students (Mage $=$ $19.04 \pm 1.04$ years) who self-reported engaging in $\leq 150$ minutes of moderate-to-vigorous intensity exercise each week. Participants were screened for non-normal vision (colourblindness), contra-indicators of performing vigorous intensity exercise using the Physical Activity Readiness Questionnaire (PAR-Q; Thomas, Reading, \& Shephard, 1992) (Appendix A), and provided informed consent. The study was reviewed and approved by an institutional research ethics board prior to any recruitment and data collection. Participants received either a $\$ 20$ honorarium or partial course credit (if recruited through the academic institution's Introductory Psychology course) for their contributions to the study.

\section{Sample Size Calculation}

Using statistical software (G*Power; Faul, Erdfeller, Lang \& Buchner, 2007) sample size for the experiment was calculated a-priori for the effect of mental fatigue on exercise decisionmaking. Results from Iodice et al. (2017) demonstrated a medium-large effect size of $\left(\eta_{\mathrm{P}}{ }^{2}=\right.$ 0.30) of physical fatigue on exercise decision-making. Using this effect size, translated to a Cohen's $w$ of .55 , and setting an alpha of 0.05 , power of 0.80 , and one degree of freedom yielded a sample size estimate of 27 for a design utilizing a chi-square goodness-of-fit test. For a mediation analysis involving a sequential multiple regression model with a medium-large effect size $\left(\eta_{\mathrm{P}}^{2}=0.30\right.$ converted to a Cohen's $f$ of .65$)$, alpha of 0.05 , power of 0.80 , and two predictors yielded a sample size estimate of 27 . Given, the sample size-estimate was considerably smaller than other estimates for regression analyses, which recommend an $N$ of $50+8(x)$, when $x=$ the 
M. Sc. Thesis - Sheereen Harris; McMaster University - Kinesiology

number of predictor variables (Green, 1991); in order to protect against a Type II error, the sample size was doubled.

\section{Tasks and Apparatus}

\section{Modified Graded Exercise Test (M-GXT)}

All participants completed a modified graded exercise test (M-GXT) protocol on a cycle ergometer (Lode Corival, Groningen, The Netherlands). The seat and handlebars were adjusted for individual comfort. Participants were told that the purpose of the task was to familiarize them with a specific range of intensities that they would need to think about later on in the study. The protocol started with a three-minute warm-up at a fixed resistance of 50 Watts (W) followed by a progressive ramp involving incremental increases of workload of one $\mathrm{W}$ every three seconds. Participants were instructed to maintain a cadence above 50 RPM, and to inform the experimenter whenever their rating of perceived exertion (RPE) increased by one unit on Borg's (1998) CR-10 scale. The target range of interest, which was relevant to the next phase of the study, was between a RPE of 3 (verbal anchor of "moderate”) and a RPE of 6 (between the verbal anchors of "strong" and "very strong”). Participants were provided with an illustration highlighting this range (See Figure 1). Once within the target RPE range, participants were instructed to focus on the sensations they were experiencing while cycling, such as: how heavy they were breathing, the tension in their muscles, and overall how it felt to exercise. After reaching the upper limit of the target range and reporting an RPE $\geq 7$, the M-GXT was terminated and the experimenter adjusted the workload to $50 \mathrm{~W}$, at which participants continued to pedal for two minutes as a cool-down. 


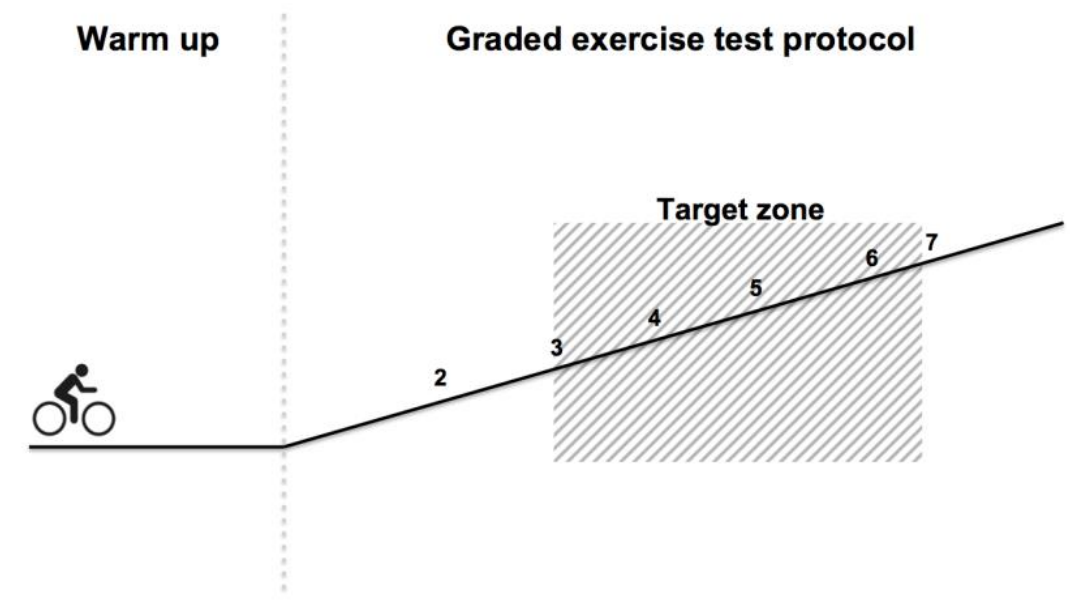

Time

Figure 1. This illustration was placed in front of participants for the duration of their modified graded exercise test (M-GXT), highlighting the "target zone" containing the RPE range of interest.

\section{Exercise Choice Protocol}

Participants were given a choice between an exercise task and a non-exercise task (see Study Procedures). Participants who selected the exercise option completed a 20-minute bout of self-paced, moderate-to-vigorous intensity exercise on the cycle ergometer. The protocol started with a two-minute warm-up at a fixed workload of $50 \mathrm{~W}$. Following the warm-up, the experimenter switched the ergometer from terminal mode (computer-defined) to manual mode (user-defined) and the experimenter set the ergometer's starting workload to the same intensity as the warm-up $(50 \mathrm{~W})$. Participants were then instructed to pedal between 60-100 RPM and to adjust the workload on the bike until they were cycling at an intensity within the target range (RPE between 3 and 6). Upon verbal confirmation that the participant was within this target 
range, the experimenter recorded a baseline value for RPE and the 20-minutes of self-paced cycling began. For the duration of the protocol participants were allowed to continually adjust their workload in order to maintain RPE within the target range. RPE was measured every two minutes to ensure compliance with these instructions. If participants reported a RPE outside of the target range, the experimenter instructed the participant to adjust the workload until they were within the target RPE range. Participants were blinded to time elapsed and workload throughout the exercise protocol. There was no verbal or motivational encouragement provided by the experimenter.

\section{Non-exercise Choice Protocol}

Participants who selected the non-exercise option completed 20-minutes of seated, nonexercise behaviour within the lab. During this time, participants were permitted to use their personal devices (e.g., smartphone, laptop computer), a laptop computer in the lab, or read from a selection of magazines or newspapers available in the lab. At the beginning of the nonexercise session, the experimenter recorded a baseline value for RPE and RPE was measured at two-minutes intervals throughout the 20-minute task, which were the only interactions between participants and the experimenter.

\section{Experimental Manipulations}

\section{High Cognitive Control Task}

Participants in the high cognitive control condition performed a task requiring high levels of cognitive control using a computerized version of the incongruent Stroop colour word task (Stroop, 1935). The task was programmed using PresentationTM software (Version 17.0; NBS 
Www.neurobs.com) and consisted of five, 2-minute blocks of 135 trials (900 milliseconds per trial), separated by 30-second breaks during which participants rated mental fatigue and mental exertion, for a total duration of 12 minutes. The colour-word stimuli were presented on a 17" flat screen computer monitor for 800 milliseconds on a white background in 48-size, Times New Roman font, followed by a 100 millisecond inter-trial interval in which the screen was blank. Participants were instructed to respond as quickly and accurately as possible to each stimulus by saying aloud the colour of the font that the word was printed in and ignoring the semantic meaning of the word (e.g., for the word "black" printed in "red" ink, the correct response was to say aloud the word "red"). This task has previously been used and reliably shown to induce high levels of mental fatigue (e.g., Brown \& Bray, 2017a; Shortz \& Mehta, 2017; Vrijkotte et al., 2018).

\section{Low Cognitive Control Task}

Participants in the low cognitive control condition watched a documentary video titled: "Planet Earth: Fresh Water", consisting of five, 2-minute sequences, separated by 30-second breaks during which participants rated mental fatigue and mental exertion, for a total duration of 12 minutes. During the task, participants were instructed to monitor the audio content for every time they heard the word "water". This task has been shown to be affectively-neutral and to maintain levels of mental fatigue (e.g., Zering, Brown, Graham, \& Bray, 2016).

\section{Measures}

\section{Ratings of perceived exertion (RPE)}


Ratings of perceived exertion (RPE) were measured using Borg's (1998) CR-10 scale ranging from 0 (no exertion at all) to 10 (maximal exertion) (See Appendix B). Participants were educated on providing RPEs using Borg's instructions (Borg, 1998, p. 47) and were provided the opportunity to ask the experimenter any questions for clarification.

\section{Ratings of perceived mental exertion (RPE-M)}

Participants provided ratings of perceived mental exertion (RPE-M) using Borg's (1998) CR-10 scale ranging from 0 (no exertion at all) to 10 (maximal exertion) (See Appendix C). This scale has been previously used in studies examining self-control depletion and exercise performance (Bray, Graham, Martin Ginis, \& Hicks, 2012; Graham \& Bray, 2015).

\section{Subjective Mental Fatigue}

A Visual Analogue Scale (VAS; Wewers \& Lowre, 1990) was used to assess mental fatigue. Participants were instructed to: "Please mark $(X)$ on the line at the point that you feel represents your perception of your current state of mental fatigue”. The response continuum consisted of a 100-millimetre line with the anchors ranging from 'none at all' to 'maximal' (See Appendix D). Scores were calculated by measuring the distance in millimetres that the ' $\mathrm{X}$ ' was placed from the left side of the scale.

\section{Moderate-to-vigorous physical activity}

Moderate-to-vigorous physical activity (MVPA) was measured using the International Physical Activity Questionnaire (IPAQ; Booth et al., 2003). The questionnaire consists of four questions asking participants to report how many days and minutes per day they spend engaging 
M. Sc. Thesis - Sheereen Harris; McMaster University - Kinesiology

in each of moderate and vigorous intensity physical activity during a typical 7-day period in the past three to five months (See Appendix E).

\section{Physical activity enjoyment}

Given findings that enjoyment of physical activity is predictive of engagement in moderate-intensity, vigorous-intensity, and total physical activity behaviours (Salmon et al., 2003), physical activity enjoyment was measured as a potential covariate. A modified version of the Physical Activity Enjoyment Scale (PACES; Kendzierski \& DeCarlo, 1991) was used to assess the extent to which participants enjoyed engaging in physical activity. The PACES consists of 18 bipolar statements scored on a 7-point scale. The wording of the original scale was altered from "Please rate how you feel at the moment about the physical activity you have been doing," to "Please rate how you feel while engaging in your preferred type of physical activity," as the original scale was created to measure an individual's enjoyment while engaging in physical activity (See Appendix F).

\section{Trait self-control}

Since the experimental manipulations required different amounts of self-control, trait self-control was measured as a potential covariate. Differences between groups on trait selfcontrol may affect the success of the experimental manipulation at inducing or maintaining levels of mental fatigue, and bias subsequent decision-making. To measure trait self-control, the 13-item Brief Self-Control Scale (Tangney, Baumeister, \& Boone, 2004) was used. Items were reported using a 5-point scale ranging from (1) not like me to (5) very much like me. An example item is "I often act without thinking through all the alternatives" (See Appendix G). 


\section{Cost-Benefit Measure}

A 2-item measure was developed by the researcher to assess each of the perceived costs and benefits of engaging in the exercise option. For the perceived costs measure, participants were presented with the statement "For the exercise option, there are:..." and responded using a 1-10 scale anchored at 1) "no disadvantages" and 10) "many disadvantages". For the perceived benefits measure, participants were presented with the statement "For the exercise option, there are:..." and responded using a 1-10 scale anchored at 1) "no advantages" and 10) "many advantages" (See Appendix H). The items were used separately as "costs" and "benefits" and as a composite measure: "benefits - costs", which was simply the reported "costs" value subtracted from the reported "benefits" value. For the ease of interpretation, results are presented as "benefits-costs".

\section{Physiological Measures}

Heart rate (HR) was measured in beats per minute (bpm) using a Polar heart rate monitor (HR monitor, Polar S625X). HR was recorded throughout the experimental session with a focus on baseline scores at the beginning of each of the cognitive control task and the 20-minute exercise choice task and at 2-minute intervals during both tasks.

\section{Study Design and Procedures}

The study employed a single-blinded, between-subjects design. Participants were stratified by sex and randomized to either the high cognitive control or low cognitive control group. In order to minimize experimenter effects, both experimenters involved in the study 


\section{Sc. Thesis - Sheereen Harris; McMaster University - Kinesiology}

followed a written script throughout the entire experimental procedure, ensuring that instructions were presented in the same manner to all participants.

Participants completed one laboratory-based testing session lasting approximately two hours. On the day prior to their testing session, participants were instructed to avoid consuming caffeine and engaging in vigorous physical activity within six hours of their testing session and to get at least eight hours of devoted rest the night before.

Upon arrival at the laboratory, participants completed the PAR-Q, provided informed consent and then completed a questionnaire assessing basic demographic information (e.g., age, sex, year of study) and the measures of MVPA, physical activity enjoyment, and trait selfcontrol. Next, anthropometric data (e.g., height [m] and weight [kg]) were recorded using a tape measure and digital scale and from those measures, body mass index (BMI) was also calculated. Participants were then fitted with a HR monitor (Polar S625X) and familiarized with the RPE scale. Next, participants mounted the cycle ergometer and completed the M-GXT protocol.

Upon completion of the M-GXT, participants dismounted the cycle ergometer and were seated at a desk for ten minutes of quiet rest to ensure that physiological arousal returned to baseline (resting HR) prior to starting the cognitive task (Byun et al., 2014). During this time, participants were instructed how to use the RPE-M scale. Following the 10-minute rest period, participants completed their respective experimental manipulation (i.e., high cognitive control vs. low cognitive control) with RPE-M, mental fatigue, and HR recorded at baseline and at 2-min intervals throughout the task.

Following the cognitive task, the experimenter informed participants that for the next part of the session they had to choose between two tasks that would both last 20 minutes. The two options involved cycling on the stationary bike for 20 minutes and maintaining their RPE within 


\section{Sc. Thesis - Sheereen Harris; McMaster University - Kinesiology}

a range between a 3 and a 6 or not exercising on the bike and completing other non-exercise tasks in the lab where they would have access to their phone, a laptop, or reading media for 20 minutes. Next, participants filled out the benefit-cost measure, rated their current level of mental fatigue and then indicated whether they would exercise or not. After making their selection, participants completed their self-selected task and upon completion, were debriefed, compensated, and thanked for their participation. Throughout the experimental procedures, the experimenter only interacted with participants to provide instructions, administer measures, and ensure the safety of participants. There was no verbal or motivational encouragement provided at any time.

\section{Data Analysis}

Descriptive statistics were computed for all study variables and the distributions of scores for each variable were tested for normality. To test for successful randomization between groups and assess potential covariates to control for in the primary analysis, independent samples $t$-tests were computed for MVPA, trait self-control, physical activity enjoyment, and BMI. Independent samples $t$-tests were computed to evaluate between-condition differences for RPE-M, mental fatigue, and HR during the cognitive experimental manipulations as well as post-cognitive task mental fatigue. A 2 (condition; high cognitive control or low cognitive control) X 2 (choice; non-exercise or exercise) contingency table (Chi-square analysis) was computed to compare frequencies of choice between conditions.

As our primary interest was to examine how varying levels of mental fatigue related to exercise choice and behaviour, Pearson's correlations were computed to examine the bivariate relationships between each of: post-cognitive task mental fatigue, perceived exercise benefits, 


\section{Sc. Thesis - Sheereen Harris; McMaster University - Kinesiology}

perceived exercise costs, perceived benefits-costs, and choice. Mental fatigue has been operationalized as the cumulative effect of a cognitive control manipulation, in line with the conceptual definition of "the feeling that people may experience after or during prolonged periods of cognitive activity" (Boksem and Tops, 2008, p.125). However, given evidence of a very strong correlation between cumulative mental fatigue and post-cognitive task mental fatigue $(r=.95$, Brown \& Bray, 2018) the operational definition in this study was post-cognitive task mental fatigue.

To assess the direct effect of mental fatigue on exercise choice, a binary logistic regression was computed. To test the assumption of a linear relation between a predictor and the logit transform of the dependent variable, a Box-Tidwell test was used. To assess our hypothesis that a cost-benefit analysis mediates the effect of mental fatigue on exercise choice, a mediation analysis was conducted using Model 4 in the PROCESS software macro (Hayes, 2017). Bootstrap procedures utilizing 10,000 simulations were computed, as recommended by Hayes and Scharkow (2013). In this analysis, a 95\% confidence interval that does not cross zero indicates a significant $(p<.05)$ indirect (mediation) effect.

For participants who chose the exercise option, self-selected exercise intensity during the task was calculated by averaging the 11 self-reported RPE values recorded during the 20 -minute exercise task to ensure compliance with task instructions (e.g., maintaining an RPE within a fixed 3-6 range). Also, the mean workload (W) for each participant was calculated by averaging the self-selected workload throughout the 20-minute exercise task. Because participants differed in the workloads performed within their individualized target exercise zones during the M-GXT, in order to normalize workload during the exercise session, the workload achieved at an RPE of 6 during the M-GXT was used as a reference value. The mean workload for each participant 
M. Sc. Thesis - Sheereen Harris; McMaster University - Kinesiology

during the exercise task was divided by the workload corresponding to each participant's RPE of 6 and multiplied by 100 to yield a relative workload score $(\% / 100)$. Separate Pearson's correlations were computed to test the association between post-cognitive task mental fatigue, mean RPE, and normalized workload performed during the 20-minute self-paced bout of exercise. All data analyses were carried out using IBM SPSS Version 20.0 (IBM, 2011). 
M. Sc. Thesis - Sheereen Harris; McMaster University - Kinesiology

\section{RESULTS}

\section{Data Screening and Assumption Checks}

Data were screened for normality using the Shapiro-Wilk test. Positively skewed variables (i.e., mental fatigue, RPE-M) were transformed using a square root transformation and the reflect and square root transformation was used for negatively skewed study variables (i.e., perceived exercise benefits). Analyses were conducted on transformed and non-transformed data and in all cases produced nearly identical results. For ease of interpretation, the results of the analyses using the non-transformed values are reported. Homogeneity of variance was assessed using Levene's test. Outliers were defined as cases with standardized scores in excess of 3.29 standard deviations from the mean (Tabachnick \& Fidell, 2001). One outlier was identified for the MVPA variable and was excluded from the $t$-test assessing differences between conditions in that variable but included in all other analyses. Mean RPE during the exercise task was screened to ensure that task instructions were followed for those selecting the exercise option. Despite repeated instructions, two participants continually reported $\mathrm{RPE}<3$ while exercising and were removed from analyses pertaining to exercise behaviours. All results are presented as $M \pm S D$.

\section{Preliminary Analyses}

Results of the independent samples $t$-tests investigating the effectiveness of the randomization procedures revealed no significant between-condition differences in MVPA ( $t(52)$ $=.12, p=.91, d=.03)$, trait self-control $(t(53)=.64, p=.52, d=.26)$, or physical activity enjoyment $(t(53)=-.37, p=.72, d=-.09)$. The low cognitive control group had significantly higher BMI $(t(53)=2.31, p=.03, d=.62)$ than the high cognitive control group. However, the means for both groups were within the normal BMI range (Center for Disease Control and 
M. Sc. Thesis - Sheereen Harris; McMaster University - Kinesiology

Prevention, 2016) and were therefore not used as a covariate in the analysis. Descriptive statistics for these variables are presented by condition in Table 1.

Table 1

Descriptive Information of Potential Covariates and Manipulation Checks by Condition

\begin{tabular}{|c|c|c|c|c|c|}
\hline & Condition & & \multirow{3}{*}{$t$} & \multirow{3}{*}{$p$} & \multirow{3}{*}{$d$} \\
\hline & $\begin{array}{l}\text { Low Cog. Control } \\
n=29 \text { (13 female })\end{array}$ & $\begin{array}{l}\text { High Cog. Control } \\
n=26 \text { (14 female })\end{array}$ & & & \\
\hline & $M \pm S D$ & $M \pm S D$ & & & \\
\hline MVPA & $150.89 \pm 108.89$ & $146.73 \pm 146.38$ & .12 & .91 & .03 \\
\hline Trait self-control & $3.36 \pm .68$ & $3.18 \pm .71$ & .64 & .52 & .26 \\
\hline PA enjoyment & $5.59 \pm .91$ & $5.67 \pm .86$ & -.37 & .72 & .09 \\
\hline BMI & $24.00 \pm 3.61$ & $21.95 \pm 2.89$ & 2.31 & .03 & .62 \\
\hline RPE-M & $1.57 \pm 1.07$ & $4.62 \pm 1.87$ & -7.32 & $<.001$ & 2.03 \\
\hline Mean mental fatigue & $22.25 \pm 17.36$ & $50.37 \pm 22.68$ & -5.19 & $<.001$ & 1.40 \\
\hline $\begin{array}{l}\text { Post cognitive task } \\
\text { mental fatigue }\end{array}$ & $24.24 \pm 20.30$ & $62.15 \pm 27.49$ & -5.86 & $<.001$ & 1.58 \\
\hline Heart rate & $88.16 \pm 11.79$ & $93.70 \pm 12.68$ & -1.66 & .10 & .45 \\
\hline
\end{tabular}

Note. $M=$ mean, $S D=$ standard deviation, $d=$ Cohen's d, MVPA = moderate-to-vigorous physical activity, RPE-M = ratings of perceived mental exertion. Mean mental fatigue $=$ Mean mental fatigue during the cognitive task. Post cognitive task mental fatigue $=$ mental fatigue reported immediately following the cognitive task. 


\section{Sc. Thesis - Sheereen Harris; McMaster University - Kinesiology}

The means for both RPE-M and mental fatigue were higher in the high cognitive control condition than the low cognitive control condition. Separate independent samples $t$-tests revealed that these differences were significant: $\operatorname{RPE}-\mathrm{M}(t(38.86)=-7.32, p<.001, d=2.03)$; mean mental fatigue $(t(53)=-5.19, p<.001, d=1.40)$. Post-cognitive task mental fatigue scores were also significantly higher in the high cognitive control condition $(t(53)=-5.86, p<.001, d=$ 1.58). There was a very strong positive relationship between mean cognitive task mental fatigue and post-cognitive task mental fatigue $(r=.95, p<.001)$ so the post-cognitive task mental fatigue score was used in the primary analysis. No differences were observed between conditions for $\operatorname{HR}(t(52)=-1.66, p=.10, d=.45)$.

\section{Primary Analyses}

Frequencies of choice between the exercise and non-exercise options are presented, by experimental group, in Figure 2. A 2 (condition) X 2 (choice) Chi-square analysis revealed no differences between conditions on the frequency of choice $\left(\chi^{2}(1)=.20, p=.66, d=.12\right.$. Frequency distributions for the post-cognitive task mental fatigue measure between conditions are presented in Figure 3. As shown in the figure, mental fatigue scores derived from the full sample were slightly positively skewed, but were within acceptable ranges for normality $($ skewness $=.50, \mathrm{SE}=.32 ;$ kurtosis $=-.91, \mathrm{SE}=.63)(\mathrm{Kim}, 2013)$. 
M. Sc. Thesis - Sheereen Harris; McMaster University - Kinesiology



- Low Cognitive Control High Cognitive Control

Option

Figure 2. Frequencies of selection of non-exercise and exercise options by condition.

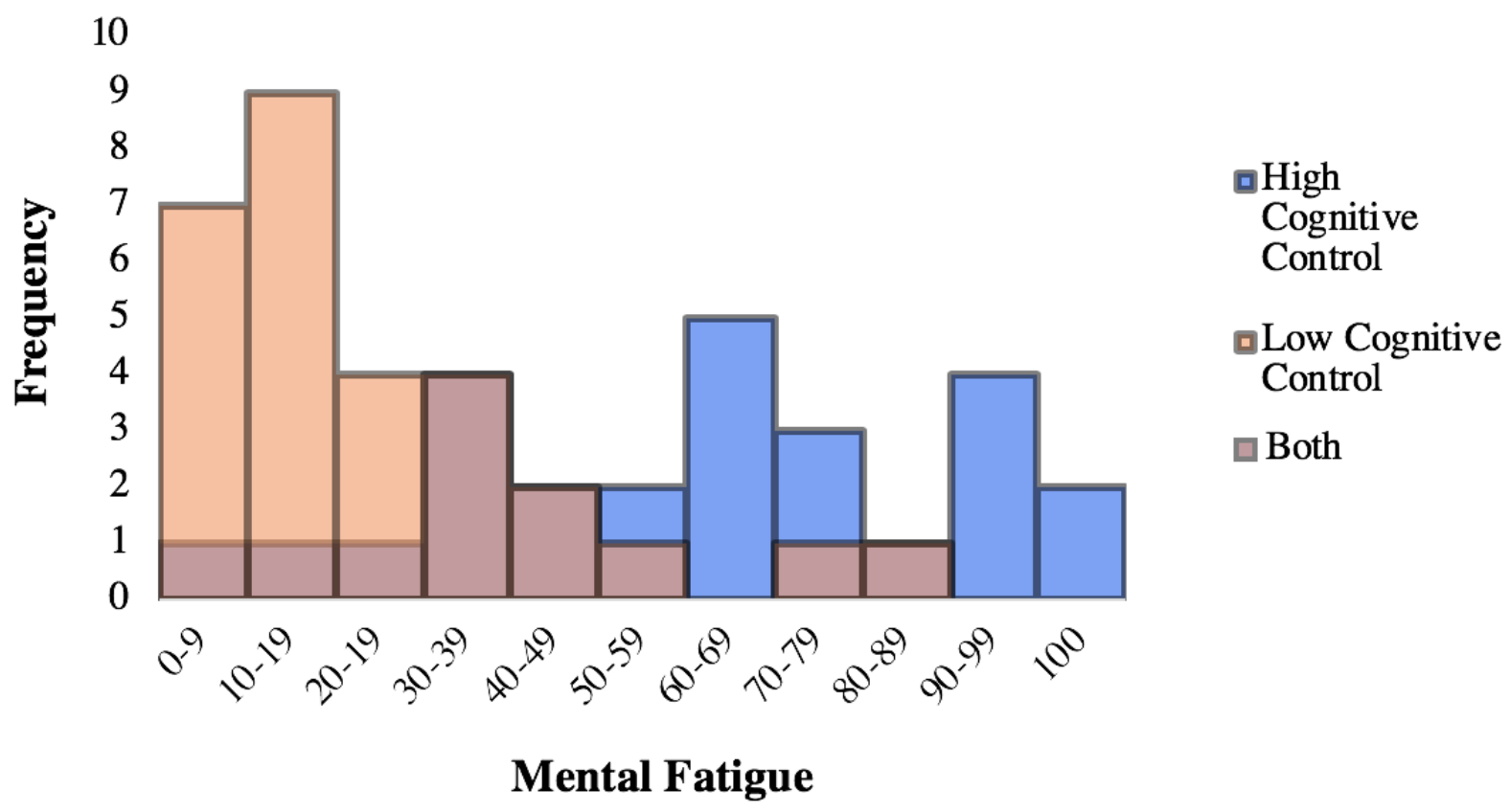

Figure 3. Frequency distributions of mental fatigue reported at the end of the cognitive task by condition (high cognitive control: blue; low cognitive control: orange; and both: purple). 
M. Sc. Thesis - Sheereen Harris; McMaster University - Kinesiology

\section{Bivariate Correlations Between Psychological Variables}

Pearson's correlations between post-cognitive task mental fatigue, perceived exercise benefits, perceived exercise costs, benefit-cost score, and choice are presented in Table 2. Small, medium, and large effects are categorized as $r$ 's $=.10, .30$, and .50, respectively (Cohen, 1988). The overall benefit-cost score was strongly correlated with both perceived exercise benefits $(r=$ $.80, p<.001)$ and costs $(r=-.78, p<.001)$; therefore the benefit-cost score was used in the primary analyses. A medium-sized effect was found between mental fatigue and the benefit-cost score $(r=-.33, p=.01)$ indicating greater mental fatigue was associated with a lower benefitcost score. A scatterplot illustrating the relationship between mental fatigue and benefit-cost score is presented in Figure 4. Additionally, a medium relationship was found between choice (coded 0 for non-exercise and 1 for exercise) and benefit-cost score $(r=.31, p=.02)$ indicating that people were more likely to choose to exercise if they perceived a higher benefit-cost score of exercising.

Table 2

Bivariate Correlations (Pearson's r), Between Mental Fatigue, Exercise Benefits, Exercise Costs, and Choice

\begin{tabular}{|c|c|c|c|c|c|c|}
\hline & $M \pm S D$ & 1 & 2 & 3 & 4 & 5 \\
\hline 1. Mental fatigue & $42.16 \pm 30.46$ & - & & & & \\
\hline 2. Exercise benefits & $7.45 \pm 2.06$ & -.18 & - & & & \\
\hline 3. Exercise costs & $3.85 \pm 2.00$ & $.35^{* *}$ & -.25 & - & & \\
\hline 4. Benefit-cost score & $3.60 \pm 3.21$ & $-.33 *$ & $.80 * *$ & $-.78 * *$ & - & \\
\hline 5. Choice $(0=$ non- & & -.12 & $.45 * *$ & -.03 & $.31 *$ & - \\
\hline
\end{tabular}


exercise, $1=$

exercise)

Note: $*=p<.05 . * *=p<.01$

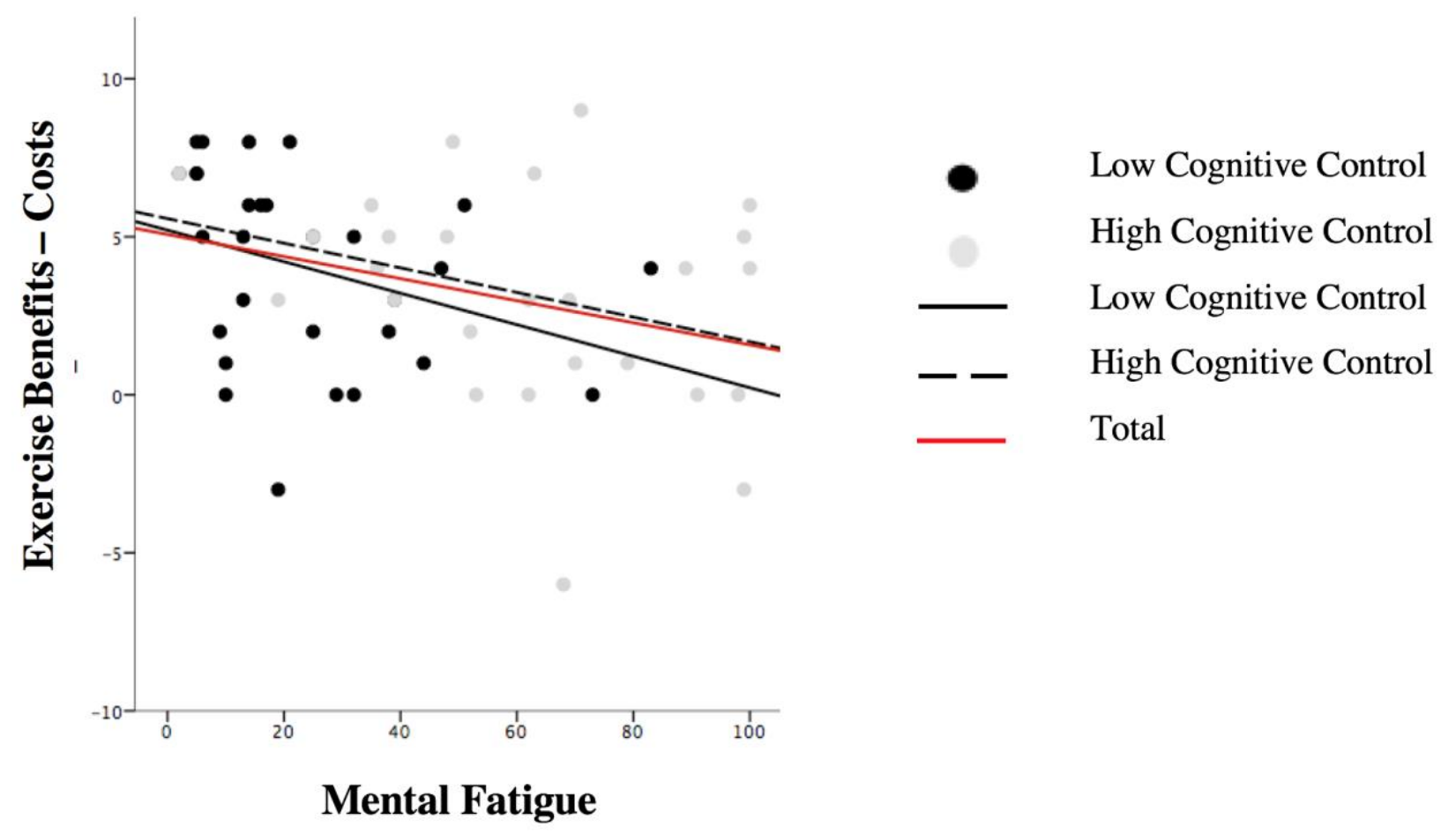

Figure 4. Scatterplot showing the relationship between subjective mental fatigue and benefit-cost analysis for participants in the high mental fatigue and low mental fatigue conditions.

\section{Indirect (Mediation) Analysis}

A Box-Tidwell test found that the interaction between mental fatigue and its natural logarithm was not statistically significant $(p=.94)$, confirming that the assumption of linearity had not been violated. The direct effect of mental fatigue on choice is presented in Figure 5. The binary logistic regression model was not statistically significant $\left(\chi^{2}(1)=.74, p=.39, d=\right.$ .23 ) and the model explained $1.8 \%$ of the variance in choice (Nagelkerke $\mathrm{R}^{2}$ ). 


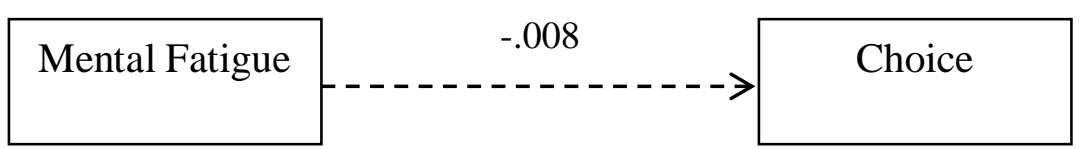

Figure 5. Direct effect of mental fatigue on exercise choice. For Choice, $0=$ non-exercise, $1=$ exercise. The coefficient is presented in unstandardized form.

In the mediation analysis, (see Figure 6), subjective mental fatigue served as the independent variable, choice as the dependent variable, and benefit-cost score as the mediator. Results revealed a significant indirect (mediation) effect of overall benefit-cost scores on exercise choice (95\% C.I. $=-.02$ to -.0004$)$.



Figure 6. Mediation analysis of the relationship between mental fatigue and exercise choice as mediated by benefit-cost score. Coefficients are presented in unstandardized form. $*=p<.05$.

\section{Mental Fatigue and Exercise Behaviour}


M. Sc. Thesis - Sheereen Harris; McMaster University - Kinesiology

In total, 30 participants (55\%) selected the exercise option. As explained above, two participants were removed from this analysis because the mean RPE reported during the exercise task was $<3$. Pearson's correlations between subjective mental fatigue, mean RPE during exercise, and percent average workload are presented in Table 3. Mean self-selected intensity ranged from 4.04 to 5.75 during the 20 -minute cycling task. There was a moderate relationship between mental fatigue and mean $\operatorname{RPE}(r=.38, p=.05)$ indicating participants who reported greater mental fatigue exercised at a greater level of exertion. There was also a small, nonsignificant, negative relationship between mental fatigue and average workload during exercise $(r=-.11, p=.59)$ suggesting greater mental fatigue was associated with less work performed.

Table 3

Bivariate Correlations (Pearson's r) Between Mental Fatigue, Exercise RPE, and Workload (W) During Exercise

\begin{tabular}{lllll}
\hline & $M \pm S D$ & 1 & 2 & 3 \\
\hline 1. Mental fatigue & $35.75 \pm 28.44$ & - & \\
2. Exercise RPE & $4.72 \pm .49$ & $.38^{*}$ & - & \\
3. Workload (\%) & $61 \pm 15$ & -.11 & .27 & - \\
\hline
\end{tabular}

Note: $N=28$ participants selecting the exercise option. $*=p<.05$. 
M. Sc. Thesis - Sheereen Harris; McMaster University - Kinesiology

\section{DISCUSSION}

The purpose of the present study was to investigate the effects of mental fatigue on people's decisions to engage in an acute bout of MVPA and whether the relationship between mental fatigue and acute decision-making is mediated by a benefit-cost evaluation. Additionally, the study aimed to examine the relationship between mental fatigue and exercise behaviours during a self-selected, self-paced, bout of exercise among those people who chose to exercise. It was found that mental fatigue did not independently predict exercise choice, however, people's benefit-cost evaluation of exercising mediated the relationship between mental fatigue and their choice of whether to exercise or not. Furthermore, higher levels of mental fatigue were associated with higher perceptions of exertion over the 20-minute cycling task.

\section{Mental fatigue and exercise decision-making}

The primary hypothesis in the present study was that mental fatigue would affect people's decisions whether to exercise or not. Contrary to predictions, mental fatigue was not independently predictive of acute exercise choice. This result indicates that mental fatigue is not likely to be the sole reason why people might choose to exercise or not, but rather may contribute to a more complex decision-making process by affecting people's evaluations of the costs and benefits of their potential actions.

These findings also raise methodological issues relating to theorized processes by which people's performance of cognitively demanding tasks can influence their exercise behaviours. Specifically, having people perform experimental manipulations involving greater or lesser cognitive demands is designed to alter characteristics of the participants, which in turn, are theorized to cause changes in behaviour or other outcomes. Thus, having one group of people 


\section{Sc. Thesis - Sheereen Harris; McMaster University - Kinesiology}

perform a cognitively-demanding task does not ensure that they will uniformly develop mental fatigue and conducting analyses only at the group level fails to account for the range of mental fatigue scores within each condition.

Following this logic, the present study examined a putative chain of processes to assess whether varying levels of mental fatigue affected choice through a causal mechanism of people's benefit-cost evaluation of engaging in exercise. Specifically, it was hypothesized that mental fatigue would lead to lower benefit-cost evaluations and that these evaluations would predict acute exercise choice. As predicted, benefit-cost evaluation mediated the effects of mental fatigue on acute exercise choice such that higher levels of mental fatigue were associated with lower benefit-cost scores, which in turn were predictive of more frequent selections of the nonexercise option. This result supports the notion that an individual's motivation for engaging in effort-based actions can be conceptualized as a trade-off between their evaluation of potential benefits and potential costs of the given task (Chong et al., 2016). Additionally, results extend other work citing the relationship between a benefit-cost analysis and people's habitual exercise/non-exercise behaviours (Marcus \& Owen, 1992), demonstrating the predictive value of a cost-benefit analysis on acute exercise choices, and the potential role of mental fatigue in the evaluation process.

\section{Mental fatigue and psychological correlates of decision-making}

Mental fatigue showed a moderate positive association with the benefit-cost evaluation, which appeared to be driven by a combination of greater perceived costs $(r=.35)$ and lesser perceived benefits $(r=-.18)$. These findings are interesting to interpret in light of previous research showing that regular exercisers reported significantly more benefits of exercise 


\section{Sc. Thesis - Sheereen Harris; McMaster University - Kinesiology}

compared with non-exercisers (Grubbs \& Carter, 2002). The high perceived benefits of exercise scores $(M=7.45 \pm 2.06$ out of 10$)$ observed in this study are reflective of a recreationally active sample or those who are in the Preparation-Maintenance stages of the TTM's stages of change. Consistent with the TTM, people who more regularly engage in exercise, have more entrenched and stable perceptions of the psychological, physical, and health benefits of exercise, which may be less responsive to momentary fluctuations in fatigue. Conversely, the perceived costs of engaging in exercise may be more susceptible to situational factors such as fatigue.

The behavioural economic principle implicating the importance of behavioural cost on choice holds particular relevance to study findings (Epstein, 1998). The present results showed that higher levels of mental fatigue were associated with larger perceived costs of exercise. One way in which exercise may be perceived as having greater costs is in terms of an increase in people's estimations of the effort requirements involved. There is consistent evidence in the literature that, when mentally fatigued, participants report greater perceptions of exertion while performing a physically-demanding task (cf. Van Cutsem et al., 2017). Therefore, when faced with a decision regarding a physical task, it seems possible that people may anticipate having to exert more effort to perform the task when they are feeling mentally-fatigued. In turn, this anticipation may increase the perceived costs of the activity. Unfortunately, anticipated effort expenditure was not assessed in this study. Future work should assess people's perceptions of anticipated effort as well as specific benefits/costs to determine what perceptions about the task (e.g., exercise) are considered in their subjective valuations of exercising.

\section{Mental fatigue and exercise behaviours}




\section{Sc. Thesis - Sheereen Harris; McMaster University - Kinesiology}

For the 28 participants who selected the exercise task, higher levels of mental fatigue were associated with higher RPE during exercise. However, neither mental fatigue nor RPE were related to how much work people performed during the 20-minute exercise bout. Collectively, these findings demonstrate that participants who were more mentally fatigued perceived the exercise bout to be more effortful despite working at the same relative workload as people who felt less fatigued. This finding appears to be inconsistent with previous studies, which have demonstrated that, when mentally fatigued, people reduce the effort they intend to invest on a subsequent physical task (Brown \& Bray, 2018; Martin Ginis \& Bray, 2010). However, given the relative work performed was similar across participants, it can be inferred that experiencing greater mental fatigue may have caused the same work to feel harder to perform. This phenomenon has been observed in several studies in the literature looking at the mental fatigue - physical performance relationship, where studies have found that when RPE is held constant during exercise, work performed is reduced (Brownsberger et al., 2013) and when work is held constant, RPE is greater (Marcora et al., 2009) when mentally fatigued.

It is also important to consider that the discrepancy between RPE and work may be partly attributable to giving participants the opportunity to opt out of exercise. As demonstrated in the Brown and Bray (2018) and Martin Ginis and Bray (2010) studies, people will plan to invest less effort on a subsequent bout of exercise when they know they have no choice but to perform the task. Since participants exercising in this study made the choice to exercise of their own volition, it may be that people who would have otherwise planned to exercise at lower intensities simply made the decision not to exercise.

The increase in RPE observed in the absence of increases in relative workload among participants who reported greater mental fatigue may have important practical implications for 


\section{Sc. Thesis - Sheereen Harris; McMaster University - Kinesiology}

physical activity behaviours people decide to pursue in their leisure time. Notably, if people are relying on internal cues such as perceived exertion while they exercise, this may lead them to believe that they are exercising at a higher intensity than they actually are. Furthermore, these increases in subjective exercise intensity may cause individuals to down-regulate the intensity at which they exercise, or potentially quit exercising sooner than when non-fatigued. To date, a number of strategies have been shown in studies to help overcome mental fatigue, such as providing autonomy supportive feedback (Graham, Bray, \& Martin Ginis, 2014), ingesting caffeine (Azevedo, Silva-Cavalcante, Gualano, Lima-Silva \& Bertuzzi, 2016), offering monetary incentives (Brown \& Bray, 2017b), and using behavioral monitoring (Brown \& Bray, under review). The success of these strategies illustrates several psychological and biologicallymediated mechanisms that can be targeted to counteract the effects of mental fatigue on physical performance, which should be investigated more fully to develop interventions to have people consistently exercise at sufficient intensities recommended for optimizing health benefits.

\section{Strengths and Limitations}

The present study addressed a critical gap by exploring a cost-benefit analysis as a potential mechanism by which mental fatigue affects exercise decision-making. A number of strengths and limitations should be noted as important considerations for interpreting the study results. Of note, the use of individual levels of mental fatigue should be viewed as a strength. It is common practice within the literature to look solely at group differences for analyzing the effects of cognitively-demanding tasks on outcomes of interest such as: time to exhaustion (Marcora et al., 2009), power output (Brownsberger et al., 2013), or intended exercise intensity (Brown \& Bray, 2018; Martin Ginis \& Bray, 2010). However, this practice assumes that 


\section{Sc. Thesis - Sheereen Harris; McMaster University - Kinesiology}

exposure to the same cognitive task results in identical responses among people, disregarding inter-individual differences. Additionally, the use of individual levels of mental fatigue allowed for a more robust test of the hypothesis that benefit-cost evaluations would explain why people would choose to exercise or not. According to Baron \& Kenny (1986), mediation depicts a causal chain between an independent and dependent variable indirectly through one or more mediating variables. In this study, it was theorized that mental fatigue would affect choice as mediated by a cost-benefit analysis. Using condition as the independent variable in this analysis would not account for the wide range of fatigue scores observed across both conditions (See Figure 3).

A further benefit to this study was having participants voluntarily sign up to participate in an exercise study. Despite the contrived nature of this study, the experimental design is illustrative of a situation many people experience. That is, they plan to engage in exercise behaviours but find themselves opting out given situational factors that present themselves. This experimental set up is also illustrative of the intention-behaviour gap (Rhodes \& de Bruijn, 2013), as participants scheduled a testing session time at their convenience, were instructed to arrive at the laboratory in their exercise attire prepared to exercise, and further provided with exercise equipment (e.g., cycle ergometer) to perform their pre-scheduled bout of exercise. Yet, despite taking these proactive steps in favour of being physically active, nearly half of the participants ultimately chose not to exercise.

The cost-benefit measure created for this study can be seen as both a strength and limitation. This measure may have had strong ecological validity as the questions did not provide specific examples of physical, health, psychological, or social factors as have been used in previous studies (Grubbs \& Carter, 2002; Lovell, El Ansari, \& Parker, 2010; Marcus \& Owen, 


\section{Sc. Thesis - Sheereen Harris; McMaster University - Kinesiology}

1992), allowing participants to consider and interpret the factors most relevant to them at that moment in time, that may not have been encompassed using a standardized set of questions. However, use of this measure can also be considered a limitation, as information obtained does not speak to specific perceived advantages or disadvantages that may be more or less related to mental fatigue or that are more predictive of decisions to exercise or not. Additionally, without assessing the validity and reliability of the measure, it is uncertain whether the questions used accurately evaluated the variables of interest or test-retest consistency of the measure.

Having a sample consisting of recreationally-active university students should be noted as a limitation as results may not be generalizable to other age groups such as children or older adults. Furthermore, given evidence that a cost-benefit analysis varies with different activity levels (Marcus \& Owen, 1992), study findings cannot be generalized to less active samples. Another limitation of the study was that mental fatigue was induced using a response inhibition task that requires cognitive control. Despite the incongruent Stroop task (Stroop, 1935) being commonly used to induce levels of mental fatigue in an experimental setting (e.g., Brown \& Bray, 2017a; Shortz \& Mehta, 2017; Vrijkotte et al., 2018), it is not possible to determine how an experimentally-derived state of mental fatigue is applicable outside of controlled, laboratory settings.

Lastly, the exercise option consisting solely of a 20-minute bout of cycling at a moderateto-vigorous intensity, alone with an experimenter in a laboratory should be recognized as a limitation. Physical activity behaviour encompasses a wide variety of contexts (e.g., continuous aerobic exercise, high intensity interval training aerobic exercise, and resistance exercise to name a few). Furthermore, people can participate alone, with others in a group, within a structured class, outdoors or indoors, listening to their favorite music, etc. In this study, participants were 
M. Sc. Thesis - Sheereen Harris; McMaster University - Kinesiology

only offered one exercise option where they participated in continuous aerobic exercise on a cycle ergometer. Given the behavioural economic principle stating that choice in part depends on the available alternatives (Epstein, 1998), it remains uncertain how decision-making may have differed if more exercise options had been available. It is possible that providing an exercise option that is more consistent with one's preferred physical activity context may alter their decision-making.

\section{Future Directions}

Some of the limitations of this study can be directly used to inform future directions, as well, the findings point to new avenues of inquiry. As previously mentioned, it is important to examine whether a cost-benefit analysis mediates the relationship between mental fatigue and exercise choice of different physical activity behaviours in different contexts including resistance exercise, HIIT exercise, and physical activity conducted with a partner, friend, or in a group setting. Research along these lines will provide a more holistic understanding of how people make decisions regarding their participation in physically active behaviours that better align with their personal preferences.

In addition to quantitatively measuring the cost-benefit analysis, inclusion of a qualitative assessment (i.e., "Which costs/benefits of physical activity are important to you when making this decision?") for the advantages and disadvantages of exercise would also provide valuable information. Given the relationship between mental fatigue and RPE during exercise found in this study, alongside the extensive body of literature citing increased perceptions of effort during physical tasks (e.g., Van Cutsem et al., 2017), it may be deduced that increases in the perceived costs resulted from increases in the anticipated effort requirements of the exercise task. 


\section{Sc. Thesis - Sheereen Harris; McMaster University - Kinesiology}

However, this hypothesis cannot be evaluated with the measures used in this study. The addition of more detailed measures of costs and benefits would allow researchers to draw meaningful conclusions regarding the strength of unique factors relevant in people's decision-making about exercise.

As noted earlier, in this sample consisting of recreationally active healthy young adults, findings are not generalizable to less active samples. Given findings that non-regular exercisers perceive the costs of exercise to be greater than its benefits (Marcus \& Owen, 1992), it seems plausible that those who are insufficiently active or sedentary will have different benefit-cost scores than those observed in this study. Consequently, a necessary first step for promoting exercise behaviours for non-exercisers may be to educate them on the benefits of being physically active in order to shift their decisional balance to favour the benefits of physical activity. Furthermore, future work should explore the decisional balance of acute exercise choice in non-exercisers and investigate whether the cost-benefit analysis of those who are sedentary or insufficiently active is similarly able to predict the decision to engage in an acute bout of physical activity.

A commonly held assumption in both economics and psychology is that effort is costly, and that when given a choice, people will learn to choose the less effortful option (Hull, 1943; Inzlicht et al., 2017). This assumption is based on other theorizing that effort is aversive (Kool \& Botvinick, 2014; Kurzban, 2016). However, it has been shown that effort can take on rewarding properties through the process known as learned industriousness (Eisenberger, 1992; Inzlicht et al., 2017). Learned industriousness involves pairing the sensation of effort with a reinforcer, which over repeated exposures, leads to the sensation of effort itself being perceived as valuable (Eisenberger, 1992). Therefore, future research should measure industriousness as a 


\section{Sc. Thesis - Sheereen Harris; McMaster University - Kinesiology}

potential moderator of people's effort-based choices (DeYoung, Quilty, \& Peterson, 2007), to test whether people with greater industriousness are more likely to choose an effortful option even when they are feeling fatigued. In this way, training learned-industriousness may serve as one approach to encourage people to be more physically active, particularly when faced with feelings of fatigue.

Importantly, future work should investigate ways in which the cost-benefit analysis can be manipulated to encourage decisions to exercise, in spite of feelings of fatigue. To date, two of the strategies that have been used to overcome mental fatigue (offering incentives and providing autonomy supportive feedback) have been used specifically in isometric handgrip exercises (Brown \& Bray, 2017b; Graham et al., 2014), while ingesting caffeine has been shown to improve cycling performance (Azevedo et al., 2016). An important consideration for interpreting these effects is that in these studies, participants had already agreed to perform a physically demanding task and performance on this task was measured. However, this provides no insight on how these strategies may factor into people's strategies for coping with feelings of fatigue when making the decision to exercise. In other words, it is important to differentiate between performance outcomes of a pre-determined bout of exercise and decisional outcomes related to one's willingness to engage in a bout of exercise that they have a choice about engaging in or not. In the future, it is necessary to identify effective strategies encouraging effortful decision-making, specifically by examining strategies for minimizing perceived costs and maximizing perceived benefits of effortful behaviours when fatigued.

\section{Conclusion}


M. Sc. Thesis - Sheereen Harris; McMaster University - Kinesiology

This study is the first to have investigated the effects of mental fatigue on acute exercise decision-making. It was found that a cost-benefit analysis mediated the relationship between mental fatigue and people's choice of whether to exercise or not. Higher levels of fatigue were associated with lower benefit-cost scores, which were in turn predictive of a reduced likelihood of choosing to exercise. Overall, the findings provide support for the use of a benefit-cost analysis on predicting acute exercise choice and the role of mental fatigue in the subjective evaluation of exercise benefits and costs. 
Appendix A. Physical Activity Readiness Questionnaire (Thomas, Reading, \& Shephard, 1992). If you answer NO to each of the following questions then you are eligible for this study:

1. Do you have a medical condition that requires you to avoid strenuous exercise?

2. Has your doctor ever said that you have a heart condition and that you should only do physical activity recommended by a doctor?

3. Do you feel pain in your chest when you do physical activity?

4. In the past month, have you had chest pain when you were not doing physical activity?

5. Do you lose balance because of dizziness or do you lose consciousness?

6. Do you have a bone or joint problem (for example, back, knee or hip) that could be made worse by a change in your physical activity?

7. Is your doctor currently prescribing drugs (for example, water pills) for your blood pressure or heart condition?

8. Do you know of any other reason why you should not do physical activity? 
M. Sc. Thesis - Sheereen Harris; McMaster University - Kinesiology

Appendix B. Ratings of Perceived Exertion (Borg, 1998).

Please report how much effort you exerted during the task.

0 Nothing at all

0.3

0.5 Extremely weak

1 Very weak

1.5

2 Weak

2.5

3 Moderate

4

5 Strong

6

7 Very Strong

8

9

10 Absolute Maximum 
M. Sc. Thesis - Sheereen Harris; McMaster University - Kinesiology

Appendix C. Ratings of Perceived Mental Exertion (Borg, 1998).

Please report how much mental effort you exerted during the task.

0 Nothing at all

0.3

0.5 Extremely weak

1 Very weak

1.5

2 Weak

2.5

3 Moderate

4

5 Strong

6

7 Very Strong

8

9

10 Absolute Maximum 
M. Sc. Thesis - Sheereen Harris; McMaster University - Kinesiology

Appendix D. Subjective Mental Fatigue Visual Analogue Scale (Wewers \& Lowre, 1990).

Please mark (X) on the line the point that you feel represents your perception of your current state of MENTAL FATIGUE.

None at all

0 100

Maximal 
Appendix E. International Physical Activity Questionnaire (Booth et al., 2003).

How active are you usually? Please consider your usual activity level during a typical 7 day period in the past 6 months and answer the following about moderate and vigorous activity participation.

\section{MODERATE Physical Activity Definition:}

Moderate physical activity or exercise includes activities such as brisk walking, light swimming, dancing, biking, gardening, and yardwork. You should be able to carry on a conversation when doing moderate activities. Please consider a TYPICAL week for you and answer the following questions about moderate activities.

1. How many days per week are you moderately physically active or do you exercise moderately? days per week

2. Approximately how many minutes are you moderately physically active or do you exercise moderately each day? minutes per day

\section{VIGOROUS Physical Activity Definition:}

Vigorous physical activity or exercise includes hard activities such as jogging, aerobics, swimming, and fast biking. You may have a hard time carrying on a conversation when doing vigorous activities. Please consider a TYPICAL week for you and answer the following questions about vigorous activities.

1. How many days per week are you vigorously active or do you exercise vigorously? days per week

2. Approximately how many minutes are you vigorously active or do you exercise vigorously each day? minutes per day 


\section{Sc. Thesis - Sheereen Harris; McMaster University - Kinesiology}

Appendix F. Physical Activity Enjoyment Scale (Kendzierski \& DeCarlo, 1991).

Please rate how you feel while engaging in your preferred type of physical activity.

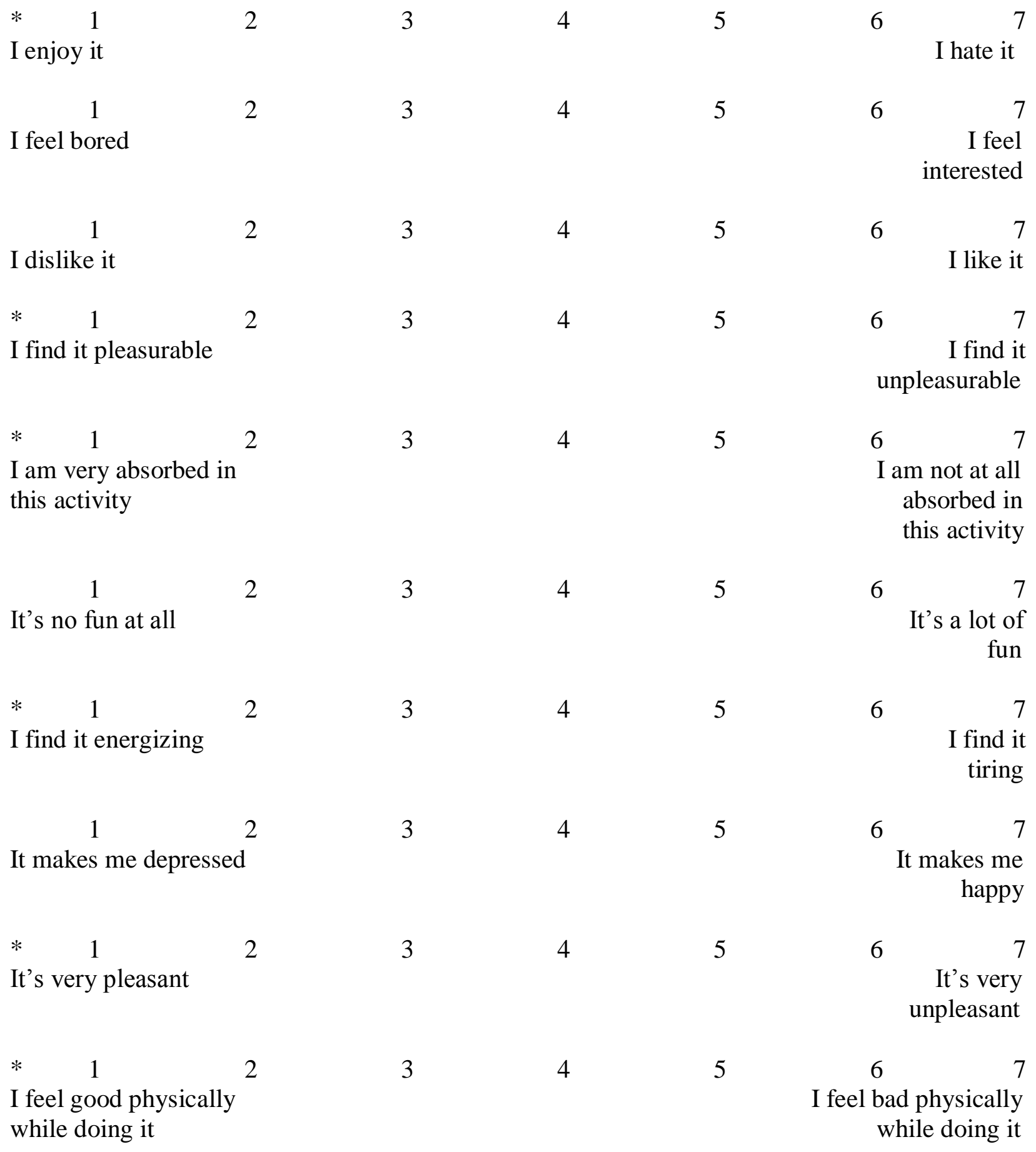


M. Sc. Thesis - Sheereen Harris; McMaster University - Kinesiology

* 1123

It's very invigorating

1
I am very frustrated

by it

* 1

2

It's very gratifying

* 12

It's very exhilarating

3

4

4

It's not at all

stimulating

* 12

It gives me a strong

sense of

accomplishment

* 112

It's very refreshing

1

2

I felt as though I would

rather be doing

something else

4

3

3

4

3

4

3

4
5

6 It's not at all invigorating

$6 \quad 7$

I am not at all frustrated by it

5

6

It's not at all gratifying

$6 \quad 7$

It's not at all exhilarating

5

6 7

It's very stimulating

5

6

7

It does not give me any sense of accomplishment at all

5

6

It's not at all refreshing

5

6 7 I felt as though there was nothing else I would rather be doing 
Appendix G. Brief Self-Control Scale (Tangney, Baumeister, \& Boone, 2004).

Please answer the following items as they apply to you. There are no right or wrong answers.

Please choose a number $(1-5)$ that best represents what you believe to be true about yourself for each question. Use the following scale to refer to how much each question is true about you.

$\begin{array}{lllll}1 & 3 & 4 & 5\end{array}$

Not at all

like me
Sometimes

like me
Very much

like me

1. I have a hard time breaking bad habits.

2. I am lazy.

3. I say inappropriate things.

4. I do certain things that are bad for me, if they are fun.

5. I refuse things that are bad for me.

6. I wish I had more self-discipline.

7. People would say that I have iron self-discipline.

8. Pleasure and fun sometimes keep me from getting work done.

9. I have trouble concentrating.

10. I am able to work effectively toward long-term goals.

11. Sometimes I can't stop myself from doing something, even if I know it's wrong.

12. I often act without thinking through all the alternatives.

13. I am good at resisting temptation. 


\section{Appendix H. Cost-Benefit Measure.}

Please answer each of the following items as they apply to you by circling the number that best represents your opinions at this moment. Please be honest, there is no right or wrong answer.

1. For the exercise option, there are:

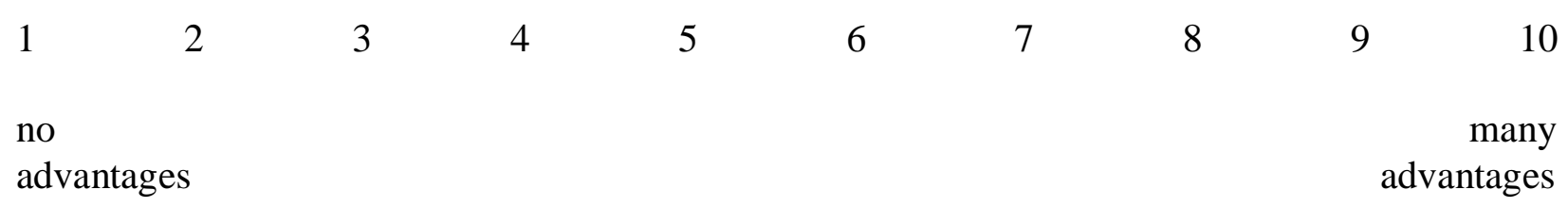

\section{For the exercise option, there are:}

$\begin{array}{lrrrrrrrr}1 & 2 & 3 & 4 & 5 & 6 & 7 & 8 & 9\end{array}$


M. Sc. Thesis - Sheereen Harris; McMaster University - Kinesiology

\section{REFERENCES}

Azevedo, R., Silva-Cavalcante, M. D., Gualano, B., Lima-Silva, A. E., \& Bertuzzi, R. (2016). Effects of caffeine ingestion on endurance performance in mentally fatigued individuals. European journal of applied physiology, 116(11-12), 2293-2303.

Baron, R. A., \& Kenny, D. A. (1986). The moderator-mediator variable distinction in social psychological research: Conceptual, strategic, and statistical considerations. Journal of Personality and Social Psychology, 51(6), 1173-1182.

Baumeister, R. F., Vohs, K. D., \& Tice, D. M. (2007). The strength model of self-control. Current directions in psychological science, 16(6), 351-355.

Berkman, E. T., Hutcherson, C. A., Livingston, J. L., Kahn, L. E., \& Inzlicht, M. (2017). Selfcontrol as value-based choice. Current Directions in Psychological Science, 26(5), 422428.

Boksem, M. A., Meijman, T. F., \& Lorist, M. M. (2006). Mental fatigue, motivation and action monitoring. Biological Psychology, 72(2), 123-132.

Boksem, M. A., \& Tops, M. (2008). Mental fatigue: costs and benefits. Brain Research Reviews, 59(1), 125-139.

Booth, M. L., Ainsworth, B. E., Pratt, M., Ekelund, U., Yngve, A., Sallis, J. F., \& Oja, P. (2003). International physical activity questionnaire: 12-country reliability and validity. Medicine \& Science in Sports \& Exercise, 195, 3508-1381.

Borg, G. (1998). Borg's perceived exertion and pain scales. Human kinetics.

Bray, S.R., Graham, J.D., Martin Ginis, K.A., \& Hicks, A.L. (2012). Cognitive task performance causes impaired maximum force production in human hand flexor muscles. Biological Psychology, 89, 195-200. 
M. Sc. Thesis - Sheereen Harris; McMaster University - Kinesiology

Brown, D. M., \& Bray, S. R. (2017a). Graded increases in cognitive control exertion reveal a threshold effect on subsequent physical performance. Sport, Exercise, and Performance Psychology, 6(4), 355-369.

Brown, D. M., \& Bray, S. R. (2017b). Effects of mental fatigue on physical endurance performance and muscle activation are attenuated by monetary incentives. Journal of Sport and Exercise Psychology, 39(6), 385-396.

Brown, D. M., \& Bray, S. R. (2018). Effects of Mental Fatigue on Exercise Intentions and Behavior. Annals of Behavioral Medicine.

Brown, D. M. Y. \& Bray, S. R.. (under review). Behavioral monitoring attenuates effects of mental fatigue on physical activity performance. Manuscript submitted to the journal Psychology of Sport and Exercise.

Brownsberger, J., Edwards, A., Crowther, R., \& Cottrell, D. (2013). Impact of mental fatigue on self-paced exercise. International journal of sports medicine, 34(12), 1029-1036.

Byun, K., Hyodo, K., Suwabe, K., Ochi, G., Sakairi, Y., Kato, M., ... \& Soya, H. (2014). Positive effect of acute mild exercise on executive function via arousal-related prefrontal activations: an fNIRS study. NeuroImage, 98, 336-345.

Camerer, C., \& Loewenstein, G. F. (2004). “'Behavioral economics: Past, present and future.' In Camerer, C., Loewenstein, G. F., Rabin, M. (Ed), Advances in Behavioral Economics (pp.1-51). New York: Russell Sage Foundation

Center for Disease Control and Prevention. (2016). Center of Disease Control and Prevention on Obesity. Retrieved from https://www.cdc.gov/obesity/adult/defining.html 
M. Sc. Thesis - Sheereen Harris; McMaster University - Kinesiology

Chong, T. J., Bonnelle, V., \& Husain, M. (2016). Quantifying motivation with effort-based decision-making paradigms in health and disease. In Progress in brain research (Vol. 229, pp. 71-100). Elsevier.

Cohen, J. (1988). Statistical power analysis for the behavioral sciences. 2nd.

Colley, R. C., Garriguet, D., Janssen, I., Craig, C. L., Clarke, J., \& Tremblay, M. S. (2011). Physical activity of Canadian adults: accelerometer results from the 2007 to 2009 Canadian Health Measures Survey (pp. 7-14). Ottawa: Statistics Canada.

DeYoung, C.G., Quilty, L.C., Peterson, J.B. (2007). Between facets and domains: 10 aspects of the big five. Journal of Personality and Social Psychology, 93(5), 880-896.

Du, W., Green, L., \& Myerson, J. (2002). Cross-cultural comparisons of discounting delayed and probabilistic rewards. The Psychological Record, 52(4), 479-492.

Eisenberger, R. (1992). Learned industriousness. Psychological Review, 99(2), 248-267.

Epstein, L. H. (1998). Integrating theoretical approaches to promote physical activity. American journal of preventative medicine, 15(4), 257-265.

Epstein, L.H. (2012). Behavioural choice theory and obesity, Virginia Tech Carilion Research Institute. Retrieved from http://research.vtc.vt.edu/videos/2012/feb/24/behavioralchoice-theory-and-obesity/.

Faul, F., Erdfelder, E., Lang, A. G., \& Buchner, A. (2007). G*Power 3: A flexible statistical power analysis program for the social, behavioral, and biomedical sciences. Behavior Research Methods, 39, 175-191.

Friese, M., Loschelder, D. D., Gieseler, K., Frankenbach, J., \& Inzlicht, M. (2018). Is ego depletion real? An analysis of arguments. Personality and Social Psychology Review, 125. 
M. Sc. Thesis - Sheereen Harris; McMaster University - Kinesiology

Gilmour, H. (2007). Physically active Canadians. Health Reports (Statistics Canada, Catalogue 82-003), 18(3), 45-65.

Graham, J. D., Bray, S. R., \& Ginis, K. A. M. (2014). "Pay the piper”: It helps initially, but motivation takes a toll on self-control. Psychology of Sport and Exercise, 15(1), 89-96.

Graham, J. D., \& Bray, S. R. (2015). Self-control strength depletion reduces self-efficacy and impairs exercise performance. Journal of Sport \& Exercise Psychology, 37, 477-488.

Graham, J.D., Martin Ginis, K.A., Bray, S.R. (2017). Exertion of self-control increases fatigue, reduces task self-efficacy, and impairs performance of resistance exercise. Sport, Exercise, and Performance Psychology, 6(1), 70-88).

Green, S. B. (1991). How many subjects does it take to do a regression analysis? Multivariate Behavioral Research, 26, 499-510.

Grubbs, L., \& Carter, J. (2002). The relationship of perceived benefits and barriers to reported exercise behaviors in college undergraduates. Family \& Community Health, 25(2), 7684.

Hagger, M. S., Wood, C., Stiff, C., \& Chatzisarantis, N. L. (2010). Ego depletion and the strength model of self-control: a meta-analysis. Psychological bulletin, 136(4), 495-525.

Hartmann, M. N., Hager, O. M., Tobler, P. N., \& Kaiser, S. (2013). Parabolic discounting of monetary rewards by physical effort. Behavioural processes, 100, 192-196.

Hayes, A. F. (2017). Introduction to mediation, moderation, and conditional process analysis: A regression-based approach. Guilford Publications.

Hayes, A. F., \& Scharkow, M. (2013). The relative trustworthiness of inferential tests of the indirect effect in statistical mediation analysis: Does method really matter? Psychological Science, 24, 1918-1927. 
M. Sc. Thesis - Sheereen Harris; McMaster University - Kinesiology

Hsu, A. S., \& Vlaev, I. (2014). Monetary cost for time spent in everyday physical activities. Social Science \& Medicine, 108, 74-80.

Hull, C. L. (1943) Principles of behavior: an introduction to behavior theory, Appleton-Century Crofts, Inc.

Inzlicht, M., \& Berkman, E. (2015). Six questions for the resource model of control (and some answers). Soc. Personal Psychol Compass, 9(10), 1-14.

Inzlicht, M., \& Marcora, S. M. (2016). The central governor model of exercise regulation teaches us precious little about the nature of mental fatigue and self-control failure. Frontiers in psychology, 7, 1-6.

Inzlicht, M., Shenhav, A., \& Olivola, C. Y. (2018). The effort paradox: Effort is both costly and valued. Trends in cognitive sciences, 22(4), 337-349.

Iodice, P., Ferrante, C., Brunetti, L., Cabib, S., Protasi, F., Walton, M. E., \& Pezzulo, G. (2017a). Fatigue modulates dopamine availability and promotes flexible choice reversals during decision making. Scientific reports, 7(535), 1-11.

Iodice, P., Calluso, C., Barca, L., Bertollo, M., Ripari, P., \& Pezzulo, G. (2017b). Fatigue increases the perception of future effort during decision making. Psychology of Sport and Exercise, 33, 150-160.

Johnson, M.W., \& Bickel, W.K. (2002). Within-subject comparison of real and hypothetical money rewards in delay discounting. Journal of the experimental analysis of behavior, $77(2), 129-146$.

Kendzierski, D., \& DeCarlo, K. J. (1991). Physical activity enjoyment scale: Two validation studies. Journal of sport and exercise psychology, 13(1), 50-64. 
M. Sc. Thesis - Sheereen Harris; McMaster University - Kinesiology

Kesaniemi, Y. A., Danforth, E., Jensen, M. D., Kopelman, P. G., LefÈbvre, P., \& Reeder, B. A. (2001). Dose-response issues concerning physical activity and health: an evidencebased symposium. Medicine \& Science in Sports \& Exercise, 33(6), S351-S358.

Kim, H.Y. (2013). Statistical notes for clinical researchers: Assessing normal distribution (2) using skewness and kurtosis. Restorative Dentistry \& Endodontics, 38(1), 52-54.

Kirby, K.., \& Maraković, N.N. (1996). Delay-discounting probabilistic rewards: Rates decrease as amounts increase. Psychonomic Bulletin \& Review, 3(1), 100-104.

Kool, W., \& Botvinick, M. (2014). A labor/leisure tradeoff in cognitive control. Journal of Experimental Psychology: General, 143(1), 131-141.

Kurzban, R. (2016). The sense of effort. Current Opinion in Psychology, 7, 67-70.

Lovell, G.P., El Ansari, W., \& Parker, J.K. (2010). Perceived exercise benefits and barriers of non-exercising female university students in the United Kingdom. Int. J. Environ. Res. Public Health, 7(3), 784-798.

MacMahon, C., Schücker, L., Hagemann, N., \& Strauss, B. (2014). Cognitive fatigue effects on physical performance during running. Journal of Sport and Exercise Psychology, 36(4), $375-381$.

Madden, G.J., Begotka, A.M., Raiff, B.R., \& Kastern, L.L. (2003). Delay discounting of real and hypothetical rewards. Experimental and Clinical Psychopharmacology, 11(2), 139-145.

Marcora, S. M., Staiano, W., \& Manning, V. (2009). Mental fatigue impairs physical performance in humans. Journal of applied physiology, 106(3), 857-864.

Marcus, B. H., \& Owen, N. (1992). Motivational readiness, self-efficacy and decision-making for exercise. Journal of applied social psychology, 22(1), 3-16. 
M. Sc. Thesis - Sheereen Harris; McMaster University - Kinesiology

Marshall, S. J., Biddle, S. J. (2001). The transtheoretical model of behavior change: a metaanalysis of applications to physical activity and exercise. Annals of behavioral medicine, 23(4) 229-246.

Martin Ginis, K. A., \& Bray, S. R. (2010). Application of the limited strength model of selfregulation to understanding exercise effort, planning and adherence. Psychology and Health, 25(10), 1147-1160.

McGuire, J. T., \& Botvinick, M. M. (2010). Prefrontal cortex, cognitive control, and the registration of decision costs. PNAS Proceedings of the National Academy of Sciences USA, 107, 7922-7926.

Mullainathan, S., \& Thaler, R. H. (2000). Behavioral economics (No. w7948). National Bureau of Economic Research.

Muraven, M., Tice, D. M., \& Baumeister, R. F. (1998). Self-control as limited resource: regulatory depletion patterns. Journal of Personality and Social Psychology, 74(3), 774789.

Ostaszewski, P., Bąbel, P., \& Swebodziński, B. (2013). Physical and cognitive effort discounting of hypothetical monetary rewards. Japanese Psychological Research, 55(4), 329-337.

Pate, R. R., Pratt, M., Blair, S. N., Haskell, W. L., Macera, C. A., Bouchard, C., ... \& Kriska, A. (1995). Physical activity and public health: a recommendation from the Centers for Disease Control and Prevention and the American College of Sports Medicine. Jama, 273(5), 402-407.

Prochaska, J. O., \& DiClemente, C. C. (1986). Toward a comprehensive model of change. In Treating addictive behaviors (pp. 3-27). Springer, Boston, MA. 
M. Sc. Thesis - Sheereen Harris; McMaster University - Kinesiology

Rhodes, R.E., \& de Bruijn, G. J. (2013). How big is the physical activity intention-behavior gap? A meta-analysis using the action control framework. British Journal of Health Psychology, 18, 296-309.

Salam, H., Marcora, S. M., \& Hopker, J. G. (2018). The effect of mental fatigue on critical power during cycling exercise. European journal of applied physiology, 118(1), 85-92.

Salmon, J., Owen, N., Crawford, D., Bauman, A., \& Sallis, J. F. (2003). Physical activity and sedentary behavior: A population-based study of barriers, enjoyment, and preference. Health Psychology, 22, 178-188.

Shortz, A. E., \& Mehta, R. K. (2017). Cognitive challenges, aging, and neuromuscular fatigue. Physiology \& behavior, 170, 19-26.

Stroop, J. R. (1935). Studies of interference in serial verbal reactions. Journal of experimental psychology, 18(6), 643-662.

Sugiwaka, H. \& Okouchi, H. (2004). Reformative self-control and discounting of reward value by delay or effort. Japanese Psychological Research, 46(1), 1-9.

Szabo, A. (2003). Acute psychological benefits of exercise performed at self-selected workloads: Implications for theory and practice. Journal of sports science \& medicine. 2(3), 77-87.

Tabachnick, B. G., \& Fidell, L. S. (2001). Using multivariate statistics (4th ed.). Boston, MA: Allyn \& Bacon

Tangney, J. P., Boone, A. L., \& Baumeister, R. F., (2004). High self-control predicts good adjustment, less pathology, better grades, and interpersonal success. Journal of personality. 72(2), 271-324.

Thomas, S., Reading, J., \& Shephard, R. J. (1992). Revision of the physical activity readiness questionnaire (PAR-Q). Canadian Journal of Sport Sciences, 17, 338-345. 
M. Sc. Thesis - Sheereen Harris; McMaster University - Kinesiology

Tremblay, M. S., Warburton, D. E., Janssen, I., Paterson, D. H., Latimer, A. E., Rhodes, R. E., ... \& Murumets, K. (2011). New Canadian physical activity guidelines. Applied physiology, nutrition, and metabolism, 36(1), 36-46.

Van Cutsem, J., Marcora, S., De Pauw, K., Bailey, S., Meeusen, R., \& Roelands, B. (2017). The effects of mental fatigue on physical performance: a systematic review. Sports Medicine, 47(8), 1569-1588.

Vrijkotte, S., Meeusen, R., Vandervaeren, C., Buyse, L., van Cutsem, J., Pattyn, N., \& Roelands, B. (2018). Mental fatigue and physical and cognitive performance during a 2-bout exercise test. International journal of sports physiology and performance, 13(4), 510516.

Wagstaff, C. R. (2014). Emotion regulation and sport performance. Journal of Sport and Exercise Psychology, 36(4), 401-412.

Wewers, M. E., \& Lowe, N. K. (1990). A critical review of visual analogue scales in the measurement of clinical phenomena. Research in nursing \& health, 13(4), 227-236.

Wichers, M., Peeters, F., Rutten, B. P., Jacobs, N., Derom, C., Thiery, E., ... \& van Os, J. (2012). A time-lagged momentary assessment study on daily life physical activity and affect. Health Psychology, 31(2), 135-144.

Zering, J. C., Brown, D. M., Graham, J. D., \& Bray, S. R. (2016). Cognitive control exertion leads to reductions in peak power output and as well as increased perceived exertion on a graded exercise test to exhaustion. Journal of sports sciences, 35(18), 1799-1807. 